\title{
Methods in Mechanical Testing of Arterial Tissue: A Review
}

\author{
Ryley A. Macrae, Karol Miller, Barry J. Doyle \\ Vascular Engineering, Intelligent Systems for Medicine Laboratory, \\ School of Mechanical and Chemical Engineering \\ The University of Western Australia, Crawley-Perth, Australia
}

\section{Corresponding author:}

Barry J. Doyle,

The University of Western Australia, 35 Stirling Highway (M050),

Crawley,

Perth, WA 6009,

Australia.

Tel: +61 864881054

Email: barry.doyle@uwa.edu.au 


\begin{abstract}
Mechanical testing of arterial tissue can provide unique insights into its behaviour. As measurement and computational techniques continue to advance, new applications can be discovered that increase the accuracy of characterising tissue behaviour. This review provides an overview of the general considerations involved in testing arterial tissue and discusses the more commonly employed in vitro techniques used to assess the mechanical properties of arterial tissue, as well as emerging techniques. The more common methods discussed are uniaxial, planar biaxial and inflation testing. With the increasing precision and versatility of optical measuring systems, and the rising interest in the mechanical behaviour of vessels with complex geometries and material properties, we also discuss the emerging trend of full-field measurement systems. Mechanical testing can be combined with bioreactor techniques to characterise the dynamic remodelling of arterial tissue in response to its mechanical environment. The analysis and characterisation provided by advanced mechanical testing techniques can produce and inform more accurate surgical simulations, as well aid in the risk prediction and modelling of pathological conditions.
\end{abstract}

Key words: Mechanical testing, experimental techniques, arterial tissue, mechanical properties.

\title{
Acknowledgements
}

The authors would like to gratefully acknowledge funding from the National Health and Medical Research Council (Grants APP1063986 and APP1083572). 


\section{Introduction}

Cardiovascular disease remains the leading cause of death in the developed world [1]. There exist many applications of mechanical engineering which are relevant to treating cardiovascular disease. Mechanical engineering techniques can help predict disease progression, guide surgical interventions and inform personalised treatment plans. An understanding of the biomechanical behaviour of vascular tissue is central to this, and in-vitro biomechanical testing can provide valuable insight. However, there are many challenges in characterising the behaviour of soft biomaterials like vascular tissue. These include their highly non-linear stress-strain relationships, large deformations under loading, potential heterogeneity and pronounced anisotropy. While simple mechanical tests can yield insight into the nature of vascular tissue, more complex methods are required to accurately characterise its behaviour, particularly in the case of samples with complex boundary conditions and irregular geometries. Similarly, methods capable of accurately assessing local mechanical behaviour and long-term responses to mechanical conditions are required to characterise the dynamic mechanical behaviour of arteries due to vascular remodelling and disease progression.

Material properties and constitutive equations for arterial tissue have a wide range of applications in the field. The ability to model the behaviour of vessels under physiological and supra-physiological loading conditions can lead to the design and optimization of therapeutical interventions, such as modelling stress produced in arterial clamping [2] and balloon dilation [3]. Similarly, data on the tensile strength of arterial tissue can be incorporated to model arterial damage and increase the accuracy of rupture risk prediction models [4-6], as well as provide models for other failure mechanisms such as artery buckling [7]. Furthermore, changes in the material properties of the wall are linked with many pathologic conditions, and can be used to characterize long term changes in arterial structure due to active vascular remodelling processes. With methods capable of assessing the elastic properties of arteries in-vivo emerging [8], relations between material properties and vascular disease could even be used to infer disease progression or arterial integrity from in-vivo measurements.

This review aims to provide a summary of the current approaches, and their relevant experimental design considerations, to quantifying the biomechanical behaviour of arterial 
tissue in-vitro. Section 2 describes some general considerations when testing arterial tissue. Section 3 examines each of the specific biomechanical test methods typically employed. Finally, in Section 4 we summarise the field and look ahead to the future.

\section{General Considerations When Testing Arterial Tissue}

When testing biological tissues, there are a number of common experimental design considerations that must be taken into account. As this paper is concerned with in-vitro biomechanical testing of arterial samples, effects peculiar to the in-vivo state such as nerve control responses, neighbouring organs and connective tissue effects will not be discussed, although their potential impact should be acknowledged when considering in-vivo behaviour.

\subsection{Human Tissue}

Vascular tissue is composed of three major layers: the intima, the media and the adventitia. The intima is the innermost layer, and is composed of an endothelial layer of cells, a subendothelial layer and an internal elastic membrane that separates the intima from the media. The elastic membrane comprises the bulk of the intima's thickness, and in larger arteries the intima can constitute a significant proportion of the artery wall's thickness. As the arteries become more peripheral, the thickness of the intimal layer decreases. Due to the thinness of the intimal layer, it frequently has a negligible effect on the solid mechanics of the artery. However, it has been shown that the intimal layer grows gradually throughout the human lifetime, and in aged humans can contribute significantly to mechanical behaviour $[9,10]$. The intimal layer is largely composed of type I collagen interspersed with smooth muscle cells. This collagen is distributed at various orientations, arranged in layers [11].

The media is composed of smooth muscle cells, elastic fibres and collagen fibres. In larger, elastic arteries - particularly the aorta - the media layer is divided by concentric lamellae of elastic fibres, between which are the smooth muscle cells and collagen fibres [12]. Smooth muscle cells in the media are circumferentially aligned [11].

The adventitial layer contains fibroblasts and fibrocytes, as well as a large degree of collagen fibrils. Collagen orientation is widely dispersed in the adventitia [11], although its mean alignment is circumferential. Due to this collagen structure, the adventitia lends considerable structural strength to the artery, particularly at high pressures where the large stretch allows 
the long collagen fibres to straighten out and bear much of the load. The adventitia is also surrounded by connective tissue, which is removed prior to biomechanical testing.

In cases where sections of the arterial vessel are excised for testing, more accurate data can be obtained via separation of the component layers and conducting individual tests upon each, in order to determine stress-strain relationships specific to each layer [13]. Fung's group [14] used bending tests to find the neutral axis location of the layered aorta and showed the variation in strain distribution across the thickness of the aorta, avoiding the need for layer separation. However, in order to assess stress-strain relationships in across the wall with common test methods, layer separation is achieved via carefully peeling each layer from the sample and separating any connective tissue. After testing, histological imaging of the samples must be conducted to ensure the sample is composed of a single arterial layer and that no significant damage has occurred, otherwise the data should be discarded.

\subsection{Animal Models}

Investigation of cardiovascular diseases employs a wide range of animal models, each of which has its own considerations in designing mechanical testing systems and protocols. Generally, the most common models of cardiovascular diseases are mouse, rat, rabbit, swine and dog animal models [15-17]. At the most basic level, test devices must be sized appropriately for the intended animal model and intended sample location. Test protocol should be designed such that it approximates in-vivo loading conditions. For simple uniaxial and biaxial testing, loading rates should correspond to the approximate physiological values, which can be inferred from heart rate and blood pressure (see table 1). In the case of inflation-extension or whole-body tests where it is desirable to reproduce in-vivo conditions, it is necessary to provide an axial stretch to the vessel. During excision, the artery will spring back to an unloaded configuration, and thus physiological stretch can be approximated via:

$\lambda_{\mathrm{i}}=\frac{l}{l_{\mathrm{o}}}$

Where $l_{\mathrm{o}}$ and $l$ are the lengths in the unloaded and loaded configurations respectively, and $i$ is the axial direction. Axial stretch will vary depending upon the subject from which the tissue sample is sourced, the type of artery and the region of that artery from which the sample was taken - for instance, Han \& Fung [18] showed that the axial stretch varied from 1.2 to 1.5 along canine and porcine aortas. Physiological data is collated in table 1 , although 
where possible data should be collected individually. Due to these differences in mechanical environments, one must take care when extrapolating from animal studies to human arteries. Similarly, only the medial and adventitial layers of animal arteries are mechanically significant, as they do not display the age related intimal thickening present in aged human arteries [9]. Animal models can provide excellent illustrative data, but one must take care with the application of constitutive parameters derived from animal tissue tests.

Table 1: physiological values of human and animal models

\begin{tabular}{llllll}
\hline Source: & Heart Rate: [19] & Pressure: & Artery: & \multicolumn{2}{l}{ Axial stretch: } \\
\hline Human & 60 & $120 / 80 \mathrm{~mm} \mathrm{Hg}$ & Aorta & $(1.12-1.30)$ & {$[20]$} \\
& & & Carotid & 1.10 & {$[21]$} \\
\hline Pig & $70-120$ & $150 / 115 \mathrm{mmHg}$ & Aorta & $1.4(1.2-1.5)$ & {$[18]$} \\
& & {$[22]$} & Carotid & 1.5 & {$[23]$} \\
& & & Renal & 1.232 & {$[24]$} \\
\hline Dog & $70-120$ & $140 / 70 \mathrm{mmHg}$ & Aorta & $1.4(1.2-1.5)$ & {$[18]$} \\
& & {$[25]$} & Carotid & 1.72 & {$[26]$} \\
\hline Rabbit & $180-350$ & $130 / 65 \mathrm{mmHg}$ & Carotid & 1.4 & {$[28]$} \\
& & {$[27]$} & & & \\
\hline Mouse & $450-750$ & $120 / 80 \mathrm{mmHg}$ & Aorta & $1.4(1-1.6)$ & {$[29]$} \\
& & {$[30]$} & Carotid & 1.8 & {$[31]$} \\
\hline
\end{tabular}

\subsection{Storage}

Between removal from the subject and testing, the sample will inevitably degrade over time, and thus samples should be tested as soon as possible to minimize the effects of autolysis. As immediate testing is not always possible, care must be taken in selecting an appropriate method for storage. Few studies have investigated the effects of refrigeration on arterial tissue - Adham et. al [32] investigated the effects of long-term (4-31 days) refrigeration on the mechanical properties of thoracic aorta and found only non-significant changes in tissue stiffness. However, other studies [33] have shown that refrigeration times between 24 to 48 hours could significantly decrease the ultimate stress and Young's modulus of aortic tissue. In the same study, Stemper et al. showed no significant difference between the mechanical behaviours of fresh tissue and that frozen in Ringer's solution for up to 3 months. Similarly, 
Ebenstein et al. [34] found no significant difference in mechanical behaviour between fresh ( $<24$ hours) and frozen samples taken from atherosclerotic plaques when subjected to nanoindentation. There is a wealth of information on the effects of cryo-preservation techniques on arterial mechanics, with many studies finding no significant change in mechanical behaviour between fresh and cryopreserved samples [32, 35-39]. Other studies have observed an increase in the stiffness of coronary arteries after freezing and cryopreservation [40, 41], although Rossett et al. only observed significant changes in coronary arteries and not femoral arteries. Conversely, an increase in sample stiffness after freezing has also been observed [42]. However, many of these studies employed small sample sizes, or the studies did not consider the effect of variance between specimens taken from the same sample or variance between animals.

More recently, O'Leary et al. [43] demonstrated that porcine aortic tissue samples can be frozen for one year without significantly affecting the mechanical behaviour. This was a large study and conducted repeated tests on tissue at time points ranging from one day to one year in order to account for errors from variance between specimens from the same animal. Whether or not this applies to human tissue needs to be investigated, however, given the similarities between porcine and human aortic tissue, it is likely that the findings apply to human tissue also. Thus, it is recommended that samples be tested within 24 hours of removal to avoid any mechanical degradation. If samples are unable to be tested within a 24 hours of excision, samples can be frozen and tested within a year with no significant effect on their mechanical properties.

\subsection{Tissue environment}

In order to more closely replicate the in-vivo environment, the tissue should ideally maintained in a bath of physiological solution such as phosphate saline buffer or KrebsRinger solution and tested at a temperature of $37{ }^{\circ} \mathrm{C}$ [44-47]. It was noted by Fung that allowing the tissue to dry resulted in a drastic increase in tissue stiffness [48], thus tissue should be kept moist whenever possible. The majority of studies have kept samples at either room temperature or maintained them at $37^{\circ} \mathrm{C}$ : as the material properties of some arterial tissue have been shown to vary with temperature $[49,50]$, maintaining arteries at physiological temperature during testing is preferable. Sample temperature must be noted if meaningful comparisons are to be made to other studies. 


\subsection{Preconditioning}

Pre-conditioning is conducted by subjecting the sample to a series of loading-unloading cycles, over which the sample demonstrates a change in stress-strain response. After a series of cycles, the loading-unloading behaviour becomes repeatable. While preconditioning is widely regarded as necessary for vascular tissue [51], a standardized maximum stretch and number of cycles for preconditioning has yet to become widely accepted. It has been shown that varying the preconditioning protocols can affect the mechanical response of many biological materials, such as porcine aortic valve [52], spinal cord [53], bovine pericardium [54] and skin [55]. The effect preconditioning has is largely dependent upon the maximum strain imposed, with larger strains during preconditioning resulting in a decrease in tissue stiffness $[53,54,56]$. Further, it was demonstrated that a long relaxation time between tests at different strain levels ( $\sim 24$ hours) resulted in a 'reset' of the material's behaviour [54]. Thus, the preconditioning protocol should be as similar to the testing protocol as possible in terms of maximum strain level and rate, so as not to influence the test data with inconsistent loading patterns. Similarly, during biaxial preconditioning the ratio between axial stretches should be kept consistent with intended test ratios. If multiple tests are to be carried out on a single specimen, then the preconditioning strain level should be the maximum strain level intended for testing. Where a maximum strain is unfeasible, such as when testing tissue until failure, the maximum preconditioning strain level should mimic physiological conditions, at the same strain rate intended for testing. A wide range in the number of preconditioning cycles have been reported in the literature, but to ensure repeatability 10 cycles should be conducted on the sample prior to testing, as 10 cycles is more than sufficient to generate repeatable data [57-60].

\subsection{Tissue Morphology}

The application of microscopy techniques to vascular tissue can yield valuable insight into the mechanisms behind tissue behaviour. After mechanical testing, a piece of tissue is removed from the specimen, fixed in formaldehyde, embedded in paraffin and sectioned for microscopy. In other cases, the specimen is imaged under the microscope during mechanical testing [61]. Various histological stains can be used to image particular biological components, with particular emphasis on collagen, elastin and smooth muscle cells. For instance, haematoxylin and eosin $(\mathrm{H} \& \mathrm{E})$ staining is used for imaging the cellular structure across the arterial wall $[9,11]$, which is useful for imaging smooth muscle cell distribution 
and orientation. Picrosirius red staining or Masson's trichrome staining is applied for imaging collagen fibres, which has been used to investigate changes in collagen alignment at different stretch ratios and directions $[6,62]$. Information on collagen fibre orientation and distribution can be incorporated into developing constitutive models for arterial tissue [26,61]. Elastin can be imaged with Verhoff staining or Miller's staining [63, 64].

These techniques can also be applied to determine morphologic changes in diseased tissue in an effort to explain changes in mechanical behaviour, such as with intraluminal thrombus [65], aneurysmal tissue [66], atherosclerotic tissue [63] or carotid plaques [67]. Similarly, changes in the morphology due to the remodelling of arterial vessels cultured as per Section 3.5 can be observed [68-71]. Microscopy is also useful for investigating the mechanism of failure in arterial tissue by investigating microstructure at the failure point, such as in calcified tissue [72] or during dissection [73].

\subsection{Constitutive Modelling}

Once data on the loading and deformation of a given tissue sample has been determined and corresponding stresses and strains calculated, a constitutive model can then be fit to the data. The elastic material properties can be characterised by a strain-energy density function $\bar{\Psi}$, such as the three-dimensional generalized Fung type model[74]:

$\bar{\Psi}=\frac{1}{2} c[\exp (Q)-1]$

$Q=b_{1} \bar{E}_{\theta \theta}^{2}+b_{2} \bar{E}_{\mathrm{zz}}^{2}+b_{3} \bar{E}_{\mathrm{rr}}^{2}+2 b_{4} \bar{E}_{\theta \theta} \bar{E}_{\mathrm{zz}}+2 b_{5} \bar{E}_{\mathrm{zz}} \bar{E}_{\mathrm{rr}}+2 b_{6} \bar{E}_{\mathrm{rr}} \bar{E}_{\theta \theta}+b_{7} \bar{E}_{\theta \mathrm{z}}^{2}+b_{8} \bar{E}_{\mathrm{rz}}^{2}+$

$b_{9} \bar{E}_{\mathrm{r} \theta}^{2}$

Where $b_{\mathrm{i}}, i=(1, \ldots, 9)$ and $c$ are non-dimensional material parameters derived from fitting the model to the data and $\overline{E_{\mathrm{IJ}}}, I, J=(r, \theta, z)$ are the Green-Lagrange strain tensor components with reference to cylindrical polar components. Due to each layer of the arterial wall having distinct mechanical properties, individual testing of each layer will yield layer-specific constitutive models which can be incorporated into two-layer (or three-layer, in the case of intimal thickening) arterial wall models. There are a variety of strain-energy functions that can be used to obtain the stress-strain relationship, both three-dimensional and twodimensional: for an overview of constitutive modelling with reference to arterial mechanics, there are multiple reviews of the field $[26,74,75]$. 


\section{Test Method}

\subsection{Uniaxial extension}

This is one of the most common tests to determine the behaviour of biological material and involves elongating a rectangular or dumbbell-shaped piece of artery wall tissue in a single direction, often until failure, and measuring the gauge length and force in the direction of elongation. The techniques used to test arterial tissue in this way have evolved from international industry standards used to test rubber [76]. Stress in a deformable solid is dependent upon the geometry, boundary conditions, material properties and the load applied to the sample. In the case of a statically determinate system - as is produced in experimental test regimes - the stress field will be independent of the material properties. The material properties can then be inferred by determining a relationship between the calculated stress and strain values. Thus in experimental testing, the geometry, boundary conditions and applied load must all be precisely known in order to determine material properties. The variables to be measured will be the initial sample dimensions, displacement during elongation and force in the direction of elongation. In addition, there are a number of factors that must be taken into account when testing arterial tissue, primarily: sample orientation, sample preparation and pre-conditioning.
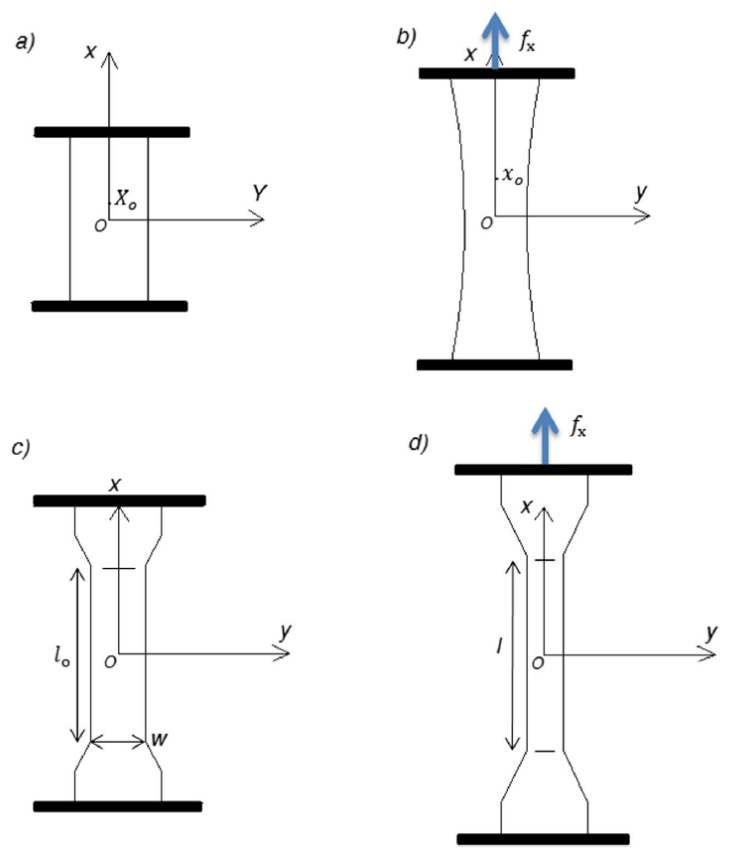

Fig. 1: Schematic of uniaixal tension test with coordinate system $O(x, y)$. a) reference configuration and $b$ ) deformed configuration with non-uniform deformation, $c$ ) reference configuration and d) deformed configuration with uniform deformation in the gauge region. 
Samples are cut into dumbbell shaped specimens of uniform width in the gauge region and their dimensions measured, either mechanically such as with callipers, or optically. The cutting is performed either by using standard dies for rubber testing [76], or cut manually such that the gage length $l_{0} \geq 5.56 \times \sqrt{\text { cross sectional area }}$ [77]. Marks are made delimiting the length of the gage region, in order for their displacement to be measured during testing. If there is insufficient tissue to produce dumbbell shaped specimens of sufficient length, then simple rectangular segments can be cut from the sample [4]. However, in this case it cannot be assumed deformation will be uniform throughout the central region and thus an alternative method of analysis is required, as is discussed below. Even if there is enough tissue for a dumbbell shaped specimen, it is recommended to perform a finite element simulation to ascertain deformation within the gage region can be assumed uniform. The average of at least three measurements of thickness and width for each specimen shall be used to calculate cross-sectional area. Each specimen is then mounted via flat clamps at both ends of the specimen, so as to exert a uniform force over the clamped area. Frequently the flat surface is lined with sandpaper or cardboard, as this has been demonstrated to be effective in minimising sample slippage [78]. Similarly, specially designed clamps with in-built grip can also be used. Due to the frequent anisotropy of aortic tissue, it is also necessary to note whether the tissue strip is longitudinally or circumferentially oriented. The tissue is then preconditioned as described in Section 2.4, until repeatable mechanical behaviour is observed.

After pre-conditioning, the sample is then subjected to elongation at a constant rate to a maximum strain, or until failure. Although the elastic behaviour of arterial tissue appears to be insensitive to strain rate over physiologic values [54], the rate at which loading is applied should be recorded. Strain rate should be chosen depending upon the aim of the experiment, and should mimic the behaviour that is to be simulated [79]. Displacements are then measured, either by taking the displacement of the machine head or gage region markers, or optically via marker tracking [80] or digital image correlation techniques [81, 82]. In processing the data, there emerge two cases - those specimens which are not of sufficient length such that deformation cannot be assumed uniform as shown in figure $1 a$ ) and b), and those in which deformation can be assumed uniform within the gage region, as shown in figure $1 \mathrm{c}$ ) and d). 


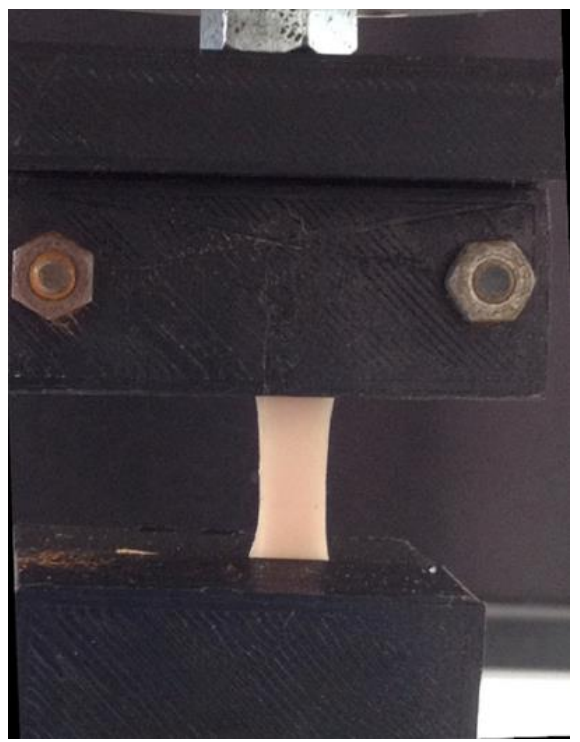

Fig. 2: Photo of a rectangular sample demonstrating non-uniform deformation under tension.

The stretch ratio $\lambda_{\mathrm{x}}$ is defined as the derivative of position $x_{\mathrm{o}}$ with respect to the reference position $X_{\mathrm{o}}$, along the $\mathrm{x}$ axis:

$\lambda_{\mathrm{x}}=\frac{d x_{\mathrm{o}}}{d X_{\mathrm{o}}}$

Thus in the case of uniform uniaxial deformation, the stretch ratio can be approximated via equation (1).

Strain along the axis of elongation can be calculated as Green-Lagrange strain $(E)$, defined as:

$E_{\mathrm{ii}}=\frac{1}{2}\left(\lambda_{\mathrm{i}}^{2}-1\right)$

Stress is calculated as the first Piola-Kirchoff stress (current force normalized by the reference (undeformed) geometry, $P$; often called the 'engineering stress') or the Cauchy stress (the current force normalized by the current (deformed) geometry, $\sigma$; often called the 'true stress'). In the case of uniform deformation, these stresses are calculated via:

$P_{\mathrm{x}}=\frac{f_{\mathrm{x}}}{t w}$

$\sigma_{\mathrm{x}}=\frac{f_{\mathrm{x}} \lambda_{\mathrm{x}}}{t w}$

Where $f_{\mathrm{X}}$ is the applied load, $t$ and $w$ are the reference thickness and width of the specimen in the gage region, and equation (7) is derived from the incompressibility of vascular tissue [83]. 
In the case of specimens where the gage region is not of sufficient length, deformation can no longer be assumed uniform (see fig. 1 a), b)). In such cases, a more complex method of assessing strain than a simple gage length ratio is required. As the material's planes perpendicular to the axis of elongation do not remain plane during deformation $[84,85]$, there exists no simple relation between machine head displacement and the stretch ratio in the direction of elongation. Hence, it is recommended that analysis be conducted by fitting a solution to a finite element model of the experimental setup [85]. This has the advantage of requiring all the same data as collected for specimens in which uniform deformation can be assumed, but avoids making assumptions about the deformation behaviour of the sample.

Although uniaxial test data is insufficient to obtain three-dimensional constitutive relations, it does provide comparative data between specimens, such as for assessing the relative difference between tissues from different regions or the impact of pathological conditions. Uniaxial tension testing also provides a simple method of assessing ultimate tensile strength when tissue is scarce, something more difficult in other tests due to the nature of gripping techniques (planar biaxial) or due to a requirement for large or intact specimens (inflationextension tests).

\subsubsection{Uniaxial Ring Test}

In the case of small animal models (particularly mouse and rat models) or small arteries like the coronary arteries, a ring tension test can provide an alternative to the uniaxial strip test. Test specimens are prepared by cutting the sample into small ring sections via circumferential cuts. Measurements are taken of the wall thickness and sample width for at least three points and the average used to calculate cross-sectional area. As with uniaxial testing, measurements can be taken optically or mechanically, such as with callipers. The ring is then mounted on two parallel pins or wires, through which the ring is elongated. As with uniaxial extension testing, the ring sample is pre-loaded and pre-conditioned until repeatable mechanical behaviour is evidenced and then (perhaps) loaded until failure. Generally, strain is calculated by measuring the displacement between the two pins [86, 87]. The stretch ratio in the circumferential direction is most simply approximated by:

$\lambda_{\theta}=\frac{l+\pi r_{\mathrm{w}}}{l_{\mathrm{o}}+\pi r_{\mathrm{w}}}$ 
Where $r_{\mathrm{w}}$ is the radius of the pins or wires, $l$ and $l_{\mathrm{o}}$ are the distances between the centre of the pins in the deformed and undeformed configurations, respectively. This assumes that strain throughout the ring is uniform, which is only true if bending effects and friction between the ring and pins are negligible. Thus, applying a lubricant to the pins can help mitigate experimental error. Alternatively, an optical measurement system can be employed to calculate local displacement, as with uniaxial tension strip tests [80, 88], in which case equation (4) is used. Strain is then calculated in the circumferential direction via equation (5). Stress can be approximated as per equation (6) where the cross-sectional area will be twice the cross-sectional area of one side of the ring.

a)

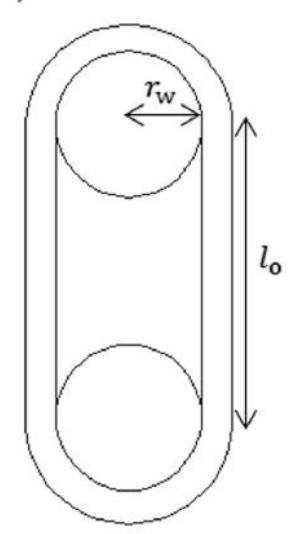

b)

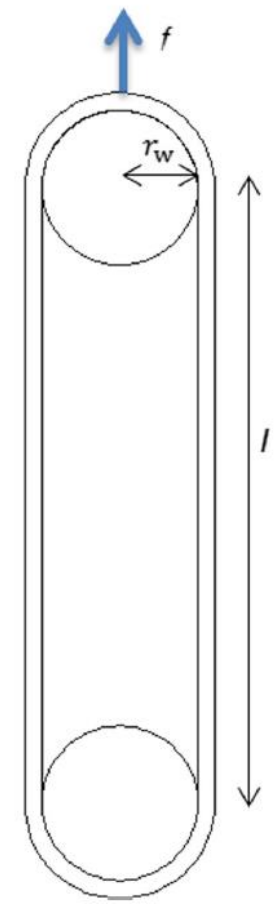

Fig. 3: Schematic of uniaixal tension test for an arterial ring, with a) reference configuration and b) deformed configuration.

Obviously ring tests require intact arterial segments and are only able to assess mechanical behaviour in the circumferential direction, and thus cannot be used to determine tissue anisotropy. This test method is not widely used in human studies as uniaxial strip tests are a more efficient means of collecting one-dimensional data; however, it is often used for small animal models due to the difficulty of preparing uniaxial strips from small tissue specimens. 


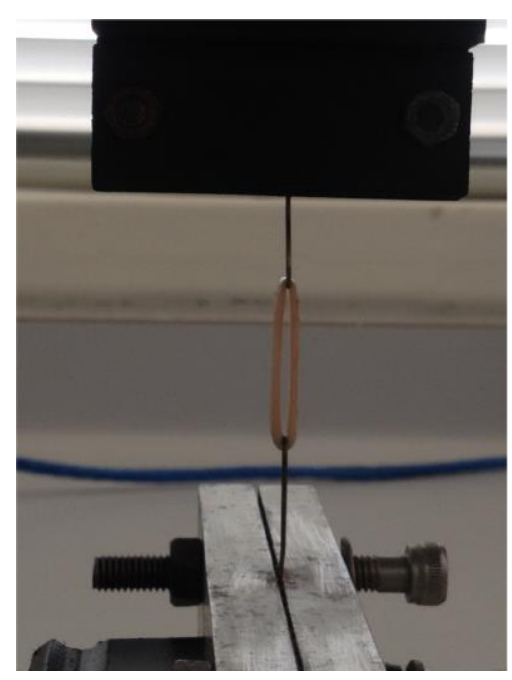

Fig. 4: Photo of an arterial ring under tension.

\subsection{Residual Strain Test}

In developing a constitutive model for the arterial wall, it is necessary to refer to the stressfree configuration of the artery. Prior to excision, the artery is under stress in two directions: an axial tension due to tethering and a circumferential tension within the unloaded arterial wall. This is evident upon cutting the artery, as when excised, the artery will spring back to its unloaded configuration. There is a similar tension in the circumferential direction that still exists when the sample is free from external loading $[89,90]$, which is evidenced by making a cut in the radial direction of an arterial vessel or ring segment. The ring will spring back to form a semi-circular segment, from which the residual strain in the circumferential direction can be derived.

Assuming that the unloaded arterial segment is in the stress-free state can lead to a significantly larger stress gradient across the vessel wall [89, 91-93], peaking at the intima. It has thus been suggested that residual strains play a role in redistributing circumferential stresses towards the load-bearing adventitia [94].

The simplest method for determining residual strains in an unloaded artery is to optically measure the opening angle $(\alpha)$ of a cross-section taken from the artery [95, 96]. As the ring is cut, it opens into a sector, on which the opening angle is defined as the angle between two radii, each drawn from the midpoint of the sector to the outer ends of the sector (see Fig. 3). Assuming no deformation in the axial direction, the circumferential stretch ratio can be calculated for a point in the vessel wall: 
$\lambda_{\theta}=\frac{\pi r}{(\pi-\alpha) R}$

$r=\sqrt{r_{\mathrm{i}}^{2}+\frac{\pi-\alpha}{\pi}\left[R_{\mathrm{i}}{ }^{2}-R^{2}\right]}$

Where $r$ and $R$ are the radii of points in the wall in the unloaded and zero-stress states respectively, and $r_{\mathrm{i}}$ and $R_{\mathrm{i}}$ the internal radii. Alternatively, circumferential stretch can be estimated via direct measurement of the deformation [29, 98, 99]:

$\lambda_{\theta}=\frac{C}{L}$

Where $C$ is the circumference in the unloaded state and $L$ the arc length of the cut specimen. In both cases, residual circumferential strain can be calculated via equation (5).

It has been shown that residual stresses also exist in the axial direction, as well as varying throughout the arterial wall $[100,101]$. Thus in order to fully characterize 3D residual stresses in the vessel wall, it is necessary to investigate residual stresses in the axial and circumferential directions in each layer of the vessel wall.

Holzapfel and co-workers [102] conducted a study wherein a circumferential ring and axial strip were excised from aortas, then glued pointwise to a cylindrical plastic tube and placed in a tissue bath to provide load-free suspension. In the case of the circumferential ring a radial cut was made as with determining opening angle, and the specimens were left in the water bath while images were taken over 16 hours. The medial layer was then removed from the adventitia, as well as the intimal from the medial layer (due to its increased thickness, and thus mechanical significance, in aged patients). Each was then glued to a cylinder and allowed to equilibrate as above, with images taken after 6 hours. Pre-stretch due to residual stresses was calculated as the ration of the initial length to unloaded length for both the inner and outer surface of the vessel wall and each of its associated layers, in both the circumferential and axial directions. These residual stresses can then be incorporated into 3D models of arterial vessels [103, 104]. 

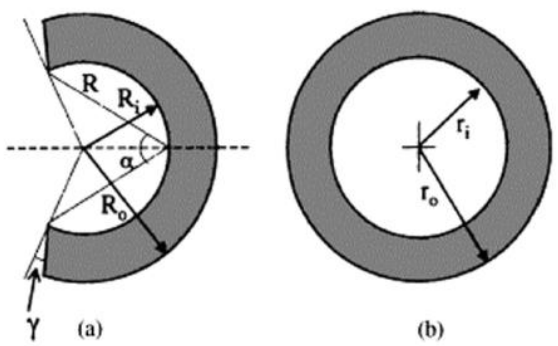

Fig. 5: Ring specimen in the a) zero stress state and b) unloaded state. Image reproduced from Rachev \& Greenwald [97]

\subsection{Planar Biaxial Test}

Uniaxial testing alone is insufficient to provide the data necessary to fully characterise the sample's mechanical behaviour. Since arterial tissue can be considered as incompressible, and occurs naturally as thin layers of tissue, planar biaxial testing provides a convenient method of assessing the mechanical properties of arterial vessels under loading regimes similar to those experienced in-vivo. The variables to be measured will be initial specimen dimensions, displacement of points during testing and axial forces.

Essentially, planar biaxial testing is conducted on a thin planar sample mounted as a flat square of tissue gripped along the edges. Squares are cut directly from the vessel wall. Initial sample dimensions are measured at this point, either mechanically or optically, as for uniaxial specimens. The sample is then mounted, either employing flat clamps [105, 106] or tethers $[58,65,107-112]$ along the edges. Clamping is conducted in a manner similar to uniaxial tension tests, wherein two flat plates are used to hold an edge in place, and sandpaper, surgical glue or ridged surfaces used to improve grip. Tethering is achieved by applying tension through a series of threads perpendicular to the edge, attached to the specimen via thin hooks or grips, which leave the specimen's edges free to expand laterally $[54,113,114]$.

While clamping the sample provides a simpler method of mounting, simulations conducted by Sun et al. [115] demonstrate that boundary effects will be greater in clamped specimens, while tethered samples produce only minimal boundary effects. Clamping can lead to very high stress concentrations at the clamped site, while reducing the central area in which edge effects are negligible. Thus, it is recommended loading be applied via tethers.

Loading is applied along each axis and is independently controlled, allowing for biaxial tests to be conducted at either constant loading rate or stretch rate whilst maintaining a constant force/stretch ratio between the axes. This also allows for investigation into the effects of 
variable ratios between the tension/stretch on material behaviour for a more complete characterisation of biaxial properties $[110-112,116,117]$. Biaxial testing can also be employed for testing until failure to examine biaxial failure mechanisms, although clamps would be required for gripping the specimen as tethers would induce failure near the gripping points. Similarly, a cruciform specimen is employed to concentrate stress in the central region. Shah et al. $[118,119]$ have employed clamped gripping of a cruciform specimen in order to conduct high-speed loading until failure. As the ultimate tensile stress will differ in the longitudinal and transverse directions [118, 120], biaxial testing is ideal for assessing ultimate stresses, although requires significantly more tissue to conduct experiments due to specimen shape.

Displacement is measured optically to avoid mechanical interference, either by tracking markers in the central region [54, 65, 110-112, 117] or a full-field method such as Electronic Speckle Pattern Interferometry (ESPI) or Digital Image Correlation (DIC) technique [58, 81, $82,116]$. Marker tracking offers the advantage of being simple and cheaper to set up, with small markers simply being glued to the specimen and imaged via camera in order to determine marker point positions. The markers must be placed so as to avoid the influence of edge effects, which conservatively will be the central $16 \%$ specimen area [115]. The area free from edge effects can be evaluated on an individual basis by assessing uniformity of the strain field as per [58, 121] or finite element modelling [115, 122].
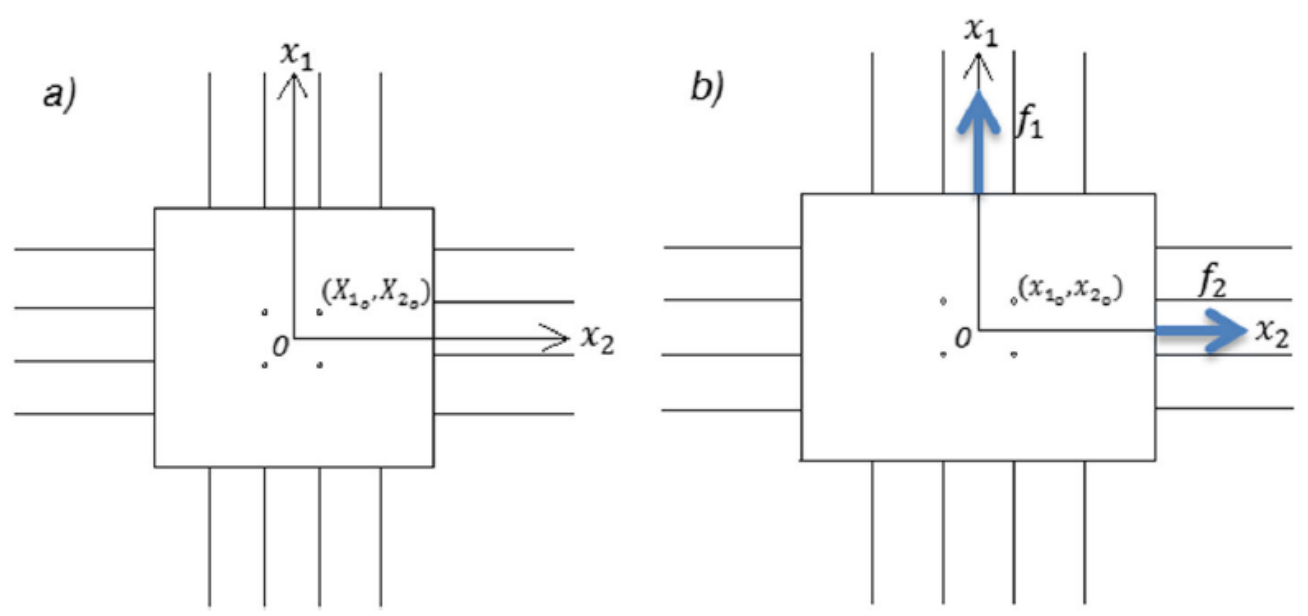

Fig. 6: Schematic of planar biaxial tension test, with coordinate system $O(x 1, x 2)$, in the a) reference and $b)$ deformed configurations. 
Full-field techniques have the advantage of being able to assess deformation locally and are thus the preferred method, with DIC offering the advantage to being insensitive to vibrations caused by the biaxial test system [123]. Either way, point positions on the specimen surface are measured and expressed as coordinates in the $\left(x_{1}, x_{2}\right)$ plane. Force is measured along the axis of applied load with a load cell.

With undeformed specimen dimensions, point positions at various time steps and corresponding axial load measurements, stress and strain values will be calculated. Subsequent equations are taken from [54]. For a given planar sample under homogenous deformation, point $\mathrm{o}\left(x_{1_{\mathrm{o}}}, x_{2_{\mathrm{o}}}, x_{3_{\mathrm{o}}}\right)$ along the $x_{1^{-}}, x_{2}$ - and $x_{3}$-axes can be approximated by:

$x_{1_{\mathrm{o}}}=\lambda_{1} X_{1_{\mathrm{o}}}+\gamma_{1} X_{2_{\mathrm{o}}}, \quad x_{2_{\mathrm{o}}}=\lambda_{2} X_{2_{\mathrm{o}}}+\gamma_{2} X_{1_{\mathrm{o}}}, \quad x_{3_{\mathrm{o}}}=\lambda_{3} X_{3_{\mathrm{o}}}$

where $x_{\mathrm{i}_{\mathrm{o}}}$ and $X_{\mathrm{i}_{\mathrm{o}}}$ are the deformed and reference coordinates respectively and $\lambda_{\mathrm{i}}$ and $\gamma_{\mathrm{i}}$ are the stretch and shear components respectively along axis $i$, with shear perpendicular to the plane of tension being negligible [54]. Thus the resulting deformation gradient tensor $\mathbf{F}$ will be:

$\boldsymbol{F}=\left[\begin{array}{ccc}\lambda_{1} & \gamma_{1} & 0 \\ \gamma_{2} & \lambda_{2} & 0 \\ 0 & 0 & \lambda_{3}\end{array}\right]$

Where due to the incompressibility constraint $\operatorname{det}(\mathbf{F})=1$,

$\lambda_{3}=\left(\lambda_{1} \lambda_{2}-\gamma_{1} \gamma_{2}\right)^{-1}$

The in-plane Green strain tensor is then calculated via:

$\boldsymbol{E}=\frac{1}{2}\left(\boldsymbol{F}^{T} \boldsymbol{F}-\boldsymbol{I}\right)$

As in many cases the shear components of deformation are negligible $[65,110-112,124]$, the relevant components of the Green Strain tensor is determined by equation (5). In general, shear components are very small compared to normal stresses and can be minimized by proper preparation of test specimens. By cutting specimens as thin as possible, utilizing at least five tethering points per side and ensuring the majority of fibres are aligned with one side of the square specimen [125], which is achieved by optically examining the specimen and assigning an average fibre direction. 

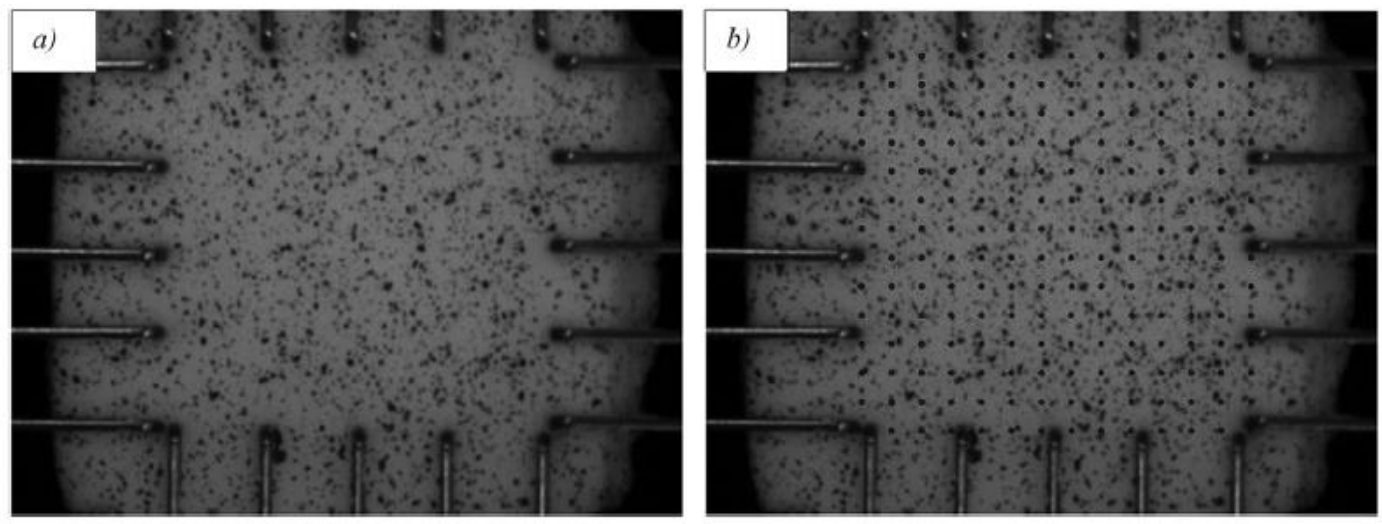

Fig. 7: a) Photo of a biaxially gripped specimen with applied speckle pattern, b) same image with virtual points for determination of deformation. Image reproduced from O'Leary et al.[58].

The first Piola-Kirchoff stress tensor components are calculated from measured load and initial specimen dimensions, where $f_{\mathrm{i}}$ is the force along axis $i, L_{\mathrm{i}}$ the initial specimen length parallel to axis $i$ and $H$ the average specimen thickness:

$P_{11}=\frac{f_{1}}{H L_{2}}, \quad P_{22}=\frac{f_{2}}{H L_{1}}$

with shear stress components and stress perpendicular to the plane assumed to be negligible [124]. The second Piola-Kirchoff stress tensor $\mathbf{S}$ and Cauchy stress tensor $\boldsymbol{\sigma}$ are calculated via:

$\boldsymbol{S}=\boldsymbol{F}^{-1} \boldsymbol{P}, \quad \boldsymbol{\sigma}=\boldsymbol{P} \boldsymbol{F}^{T}$

Thus in the case of negligible shear components and an incompressible material, Cauchy stresses can be estimated as:

$\sigma_{11}=\frac{f_{1} \lambda_{1}}{H L_{2}}, \quad \sigma_{22}=\frac{f_{2} \lambda_{2}}{H L_{1}}$

Zhang et al. [126] developed a generalized method for analysing shear components in planarbiaxial testing, as the approach described above does not produce accurate results when shear components are significant. Likewise, Sommer and co-workers [125] developed a method of quantifying shear deformations in planar biaxial extension tests. However, planar biaxial testing is insufficient to fully characterize the three-dimensional behaviour of an anisotropic material [124]. 


\subsection{Inflation testing}

More physiologically meaningful data can be obtained by preserving the native geometry of the sample and closely mimicking its in-vivo loading conditions during testing, and for this reason inflation testing is a commonly utilised test method [127]. Pressure inflation testing is an alternative method of biaxial loading, where force is applied transmurally and axially. The measured variables will be the initial sample dimensions, displacement during testing, transmural pressure and axial force, as well as torsion when twisting is incorporated. During inflation, an intact arterial vessel is secured to cannulae at both ends with suture thread or surgical glue. Any side branches are either ligated, or if small enough, can be sealed with surgical glue.

Loading is applied via stretching in the axial direction to produce axial load, pumping fluid into the vessel to produce a transmural load and in the case of multi-axial experiments, torsion applied via twisting the vessel. Pressure and force measurements are taken by transducers throughout the experiment and vessel geometry is derived through a non-contact method: frequently a CCD camera is used for marker tracking, digital image correlation or measuring vessel outer diameter. Wall thickness and undeformed internal and external diameters can be determined from separating the vessel into slices and optically measuring vessel dimensions longitudinally along the sample, or imaging the sample whole via CT scan [128].

During inflation testing, there are three loading conditions to control - vessel pressure, axial extension and torsion. The majority of experiments will keep the arterial vessel straight unless explicitly investigating the effects of tortuosity [127, 129], with only biaxial loading applied. Torsion is discussed in more detail in section 3.4.1. It is also possible to apply only uniaxial loading via inflation by attaching one end to a cannula and the other to a stopper, such that one end is sealed and the vessel is able to expand freely in the radial and axial directions $[64,130]$ 
a)

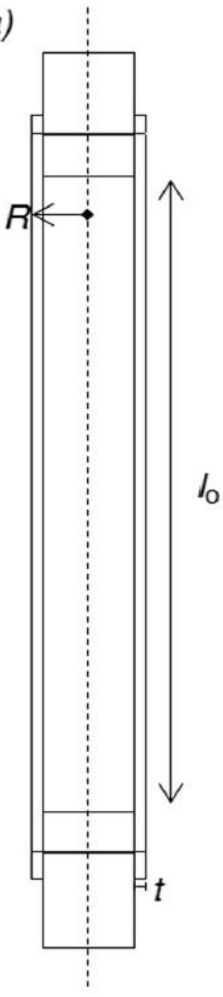

b)

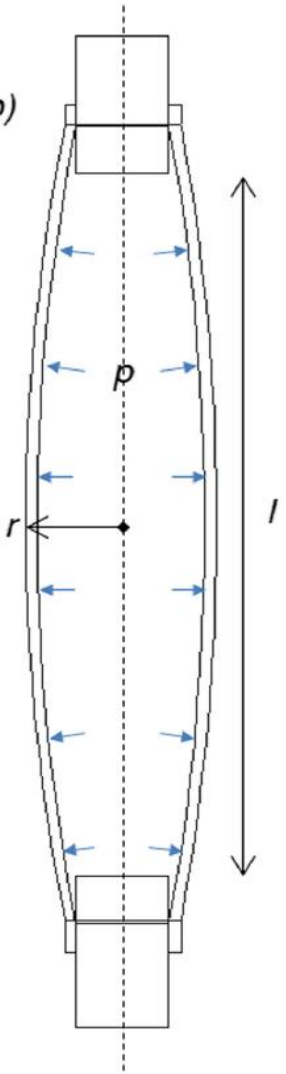

Fig. 8: Schematic of inflation-extension test for a cylindrical vessel, in a) the reference configuration and b) the deformed configuration.

As it is generally assumed that in-vivo the axial stretch and force will remain independent of internal pressure [131], experiments will frequently keep the axial stretch constant whilst varying the pressure. Axial stretches will vary from $\lambda_{\mathrm{z}}=1.0-1.6$, depending on the subject from which the tissue is sourced (see Section 2.1), as well as the type of artery and region from which the sample was taken. It is suggested that testing be conducted over a range of axial stretches in order to investigate the effect of axial stretch on the response to pressure loading, testing under no-load conditions $\left(\lambda_{\mathrm{z}}=1.0\right)$, sub-physiological, physiological and supra-physiological conditions, with physiological stretch taken from the literature (see table 1) or measured directly during excision, if possible. Pressure can be increased stepwise, taking measurements at each pressure interval $[24,132,133]$, at a constant rate $[63,134-136]$, or be controlled to mimic physiologic pressure behaviour [137, 138]. In addition to variable pressure data, the pressure can be held constant and axial stretch varied [30,61]. Position measurements are taken either as simply the diameter of the vessel, if it is assumed the vessel is a perfect cylindrical tube, and strain will be homogenous in the gage region, which is not applicable for many vessels. If local displacements are to be measured, either via marker 
tracking [139] or digital image correlation techniques, it is necessary to utilize an imaging system capable of three-dimensional analysis, as shall be discussed in section 3.4.2 [134]. Inflation testing is, of course, limited to whole vessels, and is well suited to animal studies or human autopsy studies where vessels can be excised whole, as well as tissue sourced from surgical procedures involving whole vessel extraction, such as the excision of peripheral arteries.

It is frequently assumed that for inflating arterial vessels at constant length, the specimen is cylindrical and radially inhomogeneous, and deformation can be expressed by:

$r=r(R), \quad \theta=\Theta, \quad z=\lambda_{\mathrm{z}} Z$

Where $(R, \Theta, Z)$ and $(r, \theta, z)$ are the cylindrical polar coordinates in the reference and deformed configurations, respectively. The deformed radius can be expressed as a function of the initial geometry, reference radial coordinate $R$ and axial stretch $\lambda_{\mathrm{z}}$ due to the incompressibility constraint:

$r^{2}=a^{2}+\lambda_{\mathrm{z}}^{-1}\left(R^{2}-A^{2}\right)$

Where $a$ and $A$ are the internal radii of the deformed and reference configurations, respectively [74]. Axial stretch is approximated as per equation (1), with the length being the total length of the mounted specimen. Points are either measured on the surface of the vessel or merely outer diameter is recorded. Point coordinates are taken as $(\mathrm{x}, \mathrm{z})$ and $(\mathrm{X}, \mathrm{Z})$ coordinates, where $\mathrm{x}$ and $\mathrm{z}$ are the two-dimensional coordinates provided by the imaging system, which are then mapped to their corresponding polar coordinates on the surface of the vessel. Approximating deformation in the $(\mathrm{x}, \mathrm{z})$ coordinate system as a linear polynomial and determining the deformation gradient $\mathbf{F}$ and Green strain tensor $\mathbf{E}$ as per equations (13) and (15), assuming the deformation is given by (12), will yield equation (5), with $\lambda_{\mathrm{r}}=\frac{r}{R}$ and $\lambda_{\mathrm{z}}=\frac{z}{Z}$. Under these assumptions, values for stretch will be independent of location within the gage region of the vessel, and measurements of external diameter and axial length in addition to undeformed sample geometry are sufficient to determine the strain values. Frequently, vessels are assumed to be a thin-walled vessel and thus stress calculated according to the Laplace equation $[30,135,136,140,141]$ :

$\sigma_{\theta \theta}=p\left(\frac{r}{t}-1\right)$ 
Where $p$ is the pressure and $t$ the deformed wall thickness.

However, many samples (particularly those of clinical interest) will be inhomogeneous, thick-walled, anisotropic materials, for which neither Laplace's law nor deformation of the form in (19) will hold true. As noted in [134], circumflex coronary arteries will bend and twist during inflation, introducing torsional and shear components even when the artery is kept straight. It has also been noted some arteries will experience bending and twisting invivo [142]. Furthermore, arteries can demonstrate buckling behaviour under high lumen pressure or under torsion [7]. Non-cylindrical geometries and ligated branches will also produce spatial variation of the deformation gradient. While these types of analyses are very complicated, the addition of a torsional component into pressure-inflation testing regimes can provide insight into artery behaviour under three-dimensional loading. Likewise, incorporating whole-body three-dimensional position analysis allows for spatial variation in deformations to be accounted for, which can give insight into the behaviour of heterogeneous, irregularly shaped samples.

\subsubsection{Torsion Testing}

Adding a third component to biaxial inflation-extension testing in the form of torque allows for an analysis of the shear properties of arterial vessels. The experimental setup for torsion testing is the same as for pressure-inflation tests, with the addition of a rotation motor and torque transducer at one end of the vessel. One end of the vessel is rotated by an angle $\theta$ to induce a twist in the vessel, while pressure and extension of the vessel are hold constant. This twist is repeated at variable pressures and extensions. The angle of twist $\theta$ and twist torque $T$ are recorded and used to derive the shear modulus, which has been determined for pig coronary arteries [143] and rat aortas [144]. Subsequent equations are taken from. The average shear stress in the vessel wall $\sigma_{\theta \mathrm{z}}$ will be given by [143]:

$\sigma_{\theta \mathrm{z}}=\frac{G r \theta}{L}$

Where $L$ is the vessel length, $r$ the radius, $\theta$ the current vessel twist and $G$ the shear modulus. A relation between the measured torque and the shear modulus is given by:

$$
\begin{aligned}
& T=G J \frac{\theta}{L}, \\
& J=\frac{\pi}{2}\left(r_{\mathrm{o}}^{4}-r_{\mathrm{i}}^{4}\right)
\end{aligned}
$$

Where $r_{\mathrm{o}}$ and $r_{\mathrm{i}}$ are the outer and inner radii of the vessel wall respectively. The shear modulus 
can then be incorporated into a strain-energy model: for instance, Van Epps and Vorp [145] modified a Fung-type exponential model with the inclusion of a strain parameter to account for vessels under torsion.

\subsubsection{Whole-Body Measurement}

Many clinically relevant studies involve irregular geometries, including aneurysms, stenotic arteries and areas with branching or bifurcations. In addition, while the data obtained by focusing on simplified geometries is suitable for determining material properties of a homogenous sample, the analysis of tissue with potentially heterogeneous material properties requires a method capable of determining local stresses and strains. Arterial tissue, particularly diseased tissue, can exhibit pronounced heterogeneity: for example, due to the presence of plaque [146], hypoxia due to build-up of intraluminal thrombus [66, 147] and macro or micro calcified regions of tissue [148]. Similarly, the active remodelling of vascular tissue in response to its mechanical environment can lead to local variations in material properties [149]. Thus, there is a need for methods capable of preserving native sample geometry as well as measuring local deformations.

Whole-body measurement systems utilize a combined experimental-numerical approach, wherein three-dimensional surface positions are obtained for a specimen and used to calculate strains, while wall stress is determined via computational modelling techniques. From this, the local material properties are generated via fitting a solution to the finite element simulation. Samples are excised in whole, side branches ligated and mounted upon cannulae as per inflation testing, although branched or bifurcated vessels will require specially constructed mounts that they can be set-up in their in-vivo geometries. Force measurements at the boundaries and pressure measurements are taken as for cylindrical inflation tests, and wall thickness across the specimen measured after testing by dissection and imaging of the sample.

Measurements of deformations are obtained via a non-contacting method such as a bi-plane video system, whereby marker locations are tracked in 2D images and extrapolated into 3D positions. This method has been used for obtaining regional strain fields for a wide range of vessel types, such as anterior lens capsules [150], arterial bifurcations [151] and cylindrical sections of artery from both large [139, 152] and small animals [153]. Three dimensional point tracking is used to produce deformation fields across the surface of the membrane, although this is limited to the region facing the camera system. Thus, constructing a full-body 
three-dimensional strain field requires either a multi camera setup or rotation of the sample for $360^{\circ}$ imaging. Other studies have constructed vision systems capable of panoramic imaging, allowing whole-body deformation data to be collected from a single image [154157]. Strain is determined via calculating the deformation gradient in the plane tangential to the surface. This can be accomplished by assuming deformation is homogenous and planar within the domains delimited by each triplet of markers, or by meshing the geometry obtained from DIC with triangular elements $[155,158]$. For a marker triplet $(\mathbf{A}, \mathbf{B}, \mathbf{C})$, the deformation of vectors $x_{\mathrm{AB}}$ and $x_{\mathrm{AC}}$ along the $x_{1}$ - and $x_{2}$-axes can be expressed by Equation (12), such that the 2D deformation gradient can be determined [155]:

$$
\left[\begin{array}{ll}
x_{1} \mathrm{AB} & x_{2} \mathrm{AB} \\
x_{1} \mathrm{AC} & x_{2} \mathrm{AC}
\end{array}\right]=\left[\begin{array}{ll}
F_{11} & F_{12} \\
F_{21} & F_{22}
\end{array}\right]\left[\begin{array}{ll}
X_{1} \mathrm{AB} & X_{2 \mathrm{AB}} \\
X_{1} \mathrm{AC} & X_{2 \mathrm{AC}}
\end{array}\right]
$$

The Green Strain Tensor can be calculated from equation (15).

Once the wall thickness distribution, applied loads (force and pressure) and deformations have been measured, local wall tensions and material constitutive parameters can be determined for the sample via a numerical approach. A wide variety of methods for this analysis have been reported in the literature [159]. An inverse method based on successive response surface methodology [160] was used to calculate material parameters of an aortic porcine valve, while a virtual fields method was utilised for the full-field data of a roughly cylindrical arterial segment [161]. Genovese et al. [156] used a point-wise elastostatic approach [162-164] which had previously been applied to in-vivo CT scans of aneurysms, while Joldes et al. [165] developed a Total Lagrangian based method for inverse deformation problems.

As shown by Joldes et al. [166], the stress resultants will depend only very weakly on material properties even in a thick-walled pressure loaded vessel; therefore, stress resultants can be computed simply if the deformed geometry, loading and boundary conditions are known. Given a deformed geometry, internal pressure and wall thickness, finite element analysis can be conducted on the deformed configuration with the material set as a very stiff (i.e. $\mathrm{E} \geq 10 \mathrm{MPa}$ ) linear elastic material, such that further deformation under loading conditions is negligible. Due to this very weak dependence on material properties, the verystiff material is chosen largely for convenience. While this method does not predict the 
deformation of the sample, it provides a rapid and robust method of assessing stress resultants, which can be combined with deformation data from optical methods.

Whole-body experimental-numerical methods, while complex, are capable of analysing complex geometries and anisotropic and heterogeneous material, providing significant advantages over simplified experimental approaches.

\subsubsection{Membrane Bulge}

Similar to whole-body measurement systems, a membrane-bulge test collects threedimensional geometrical data through non-contacting methods. However, whilst the wholebody measurement system preserves the native geometry of the sample, membrane-bulge testing mounts the sample as a flat planar membrane clamped firmly into the experimental system. While many other planar biaxial tests are limited in their ability to test for ultimate tensile strength due to the methods used to apply load - such as hooks - resulting in tissue failure at or near the contact points, membrane-bulge tests utilise fluid or air pressure to produce the load. This has the advantages of more closely mimicking the in-vivo loading conditions as well as allowing the sample to rupture in a manner not dependent on the method of clamping the sample. This method is thus ideal for determining ultimate tensile strength data for the sample, which is especially relevant in the case of aneurysmal tissue. In a typical membrane-bulge test, a planar sample is mounted between two clamping plates and the edges rendered air-tight. The sample is then pressurised from one side, forming a bulge in the sample. A camera system is used to collect position data across the bulge over a range of pressures and the sample is usually pressurised until failure occurs. The approach is the same as for whole-body measurement: initial thickness and dimensions are taken, applied load (pressure) and corresponding surface deformations (via marker tracking or digital image correlation) measured. Surface deformation gradients and strains are calculated as for wholebody systems and stress evaluated via a numerical method. 
a)
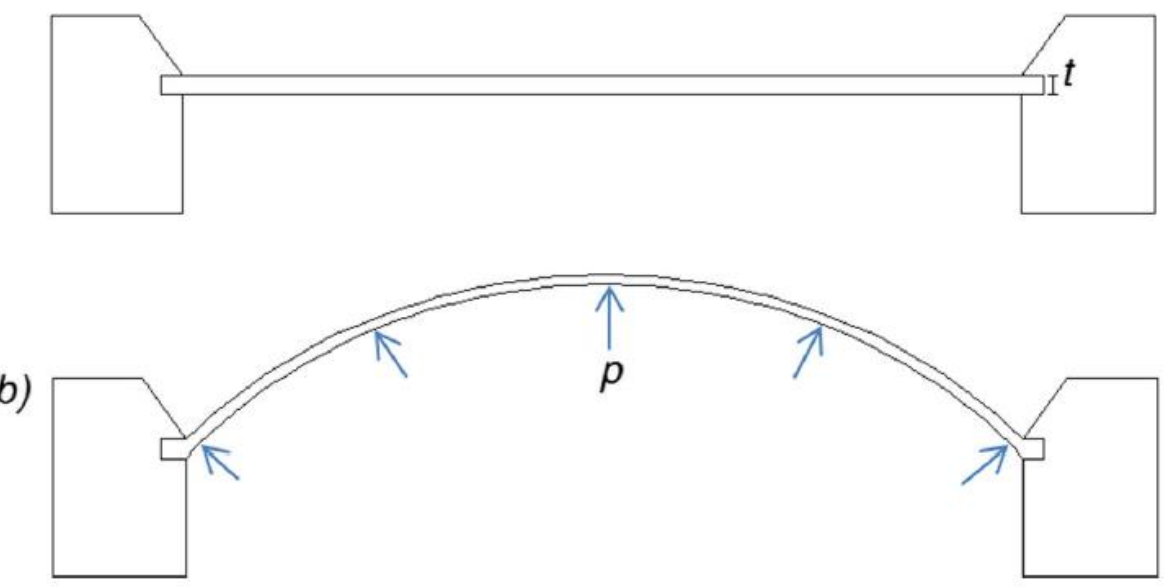

Fig. 9: Schematic of membrane bulge test for a planar sample, in the a) reference and b) deformed configurations.

The earliest membrane bulge experiments on arterial tissue were carried out by Mohan and Melvin [167] and were used to test human aortic tissue. A single camera was used to collect images of the sample, which was marked with concentric rings and changes in the diameter of these rings were used to estimate strains over the bulge. Combined with the thickness of the sample and pressure at rupture, an ultimate stress general to the sample was obtained. Marra et al. [168] utilised a similar method, analysing curvature at the pole and estimating Cauchy stresses in the axial and circumferential directions via the stress estimates provided by Janz [169]. Other methods employ biplane camera systems to provide three-dimensional data as with full-field experiments, with Hsu et al. [170] demonstrating its use on fusiform aneurysm tissue. More recent methods employ numerical methods to analyse local stress resultants and material properties, such as a virtual fields method [158] and a point-wise inverse approach $[171,172]$. These analyses have the advantage of easily allowing local stress resultants to be estimated for the rupture point directly, due to the simple planar nature of the specimen. Membrane bulge tests allow for testing that more closely mimics physiologic loading states, as well as being able to provide local stress analysis at the sample's failure point. However, this testing method does not preserve the natural geometry of the sample, which may limit its application in some cases. Similar to planar biaxial testing, this method is limited to samples large enough to be mounted and is thus unsuitable for small animal models, small human arteries or when tissue is scarce. 


\subsection{Time Dependent testing}

Benchtop testing systems can also be adapted to incorporate cell culture techniques in order to examine the long term effects of mechanical loading. It is well known that arteries respond to changes in their mechanical environment in a process known as arterial remodelling [149, 173-175] and ex vivo systems can offer many advantages in characterising this process. While many studies have examined the effects of varying global mechanical parameters in vivo, such as by inducing hypertension in animal models, ex vivo systems can allow for precise control of the pertinent mechanical factors. Additionally, where mechanical testing is concerned, organ culture systems allow for multiple mechanical tests to be conducted on the same sample, whereas in vivo models require excision of the tissue for rigorous testing. While ex vivo systems do have the drawback of not being able to precisely replicate the in vivo environment, this does have the effect of excluding biomechanical changes caused due to hormonal responses or nervous stimuli, isolating the impact of mechanical loading.

\subsubsection{Bioreactor Studies}

Organ culture systems allow for investigation into the dynamic response of arteries to imposed loading conditions, as well as preserving cells for molecular biology analysis. The three major loading conditions involved in vascular remodelling are transmural pressure, flow rate and axial extension, corresponding to the circumferential stress, wall shear stress and axial load in the sample, respectively. Thus, an effective test system must be capable of independently controlling all three factors. Early experiments involved culturing artery segments in a custom rig, where wall tension was applied via steel wires or by being cultured around a needle $[176,177]$ before being subjected to mechanical testing. As organ culturing techniques have improved over time, the same systems used to simulate in-vivo loading conditions can be used for mechanical testing of the tissue. Arterial vessels are cultured in much the same setup as cylindrical inflation tests and mechanical testing conducted via the same procedures. This allows mechanical tests to be applied to the same sample over the entire timeframe of the test, providing data of its dynamic response to altered loading conditions.

The more basic organ cultures are capable of controlling transmural pressure and fluid flow by way of pumping fluid into the vessel at a constant rate, incorporating the vessel into a flow loop [28, 178-181]. Pressure is frequently controlled by means of a reservoir and flow rate set by the pump, with both remaining constant throughout the experiment. Ideally, the flow rate 
and pressure waveform should mimic in-vivo behaviour as closely as possible and so more recent systems utilise programmable pumps to generate cyclical flow profiles. Whether the subject is animal or human, data on heart rate, blood pressure and blood flow are all readily available in the literature and can be incorporated into the system design. Thus, organ culture systems can be used to investigate the effects of varying transmural pressure ([23, 182-184] axial loading $[68,69,140]$, and shear stress $[70,71]$ on mechanical properties under conditions very similar to that in-vivo, using the same equipment as is being used to produce this mechanical environment. Maintaining this mechanical environment can also allow for investigation into the effects of culturing vessels with different serum factors $[30,185]$ or other biochemical variables, as well as being useful for both growing and testing tissueengineered constructs [186]. Combined organ culture/mechanical testing systems can play a significant role in increasing our understanding of dynamic arterial remodelling, providing valuable insight into the pathogenesis of common cardiovascular diseases.

\section{Concluding Remarks}

As the accuracy and versatility of optical measuring systems increases, it is becoming increasing common practice to evaluate strain and stress locally for specimens via techniques such as electronic speckle pattern interferometry or digital image correlation. Even in simple tests, these methods can provide information about local variances in tissue properties and avoid error from utilizing global estimates of stress and strain.

While uniaxial testing, both of ring and flat strip specimens, is unable to characterize biaxial or multiaxial behaviour and thus provides less useful information, it can provide a simple method for comparative studies of diseased and healthy arterial tissue and is necessary in cases when tissue is too scarce or samples too small to conduct other mechanical testing.

Planar biaxial testing has the advantage of better simulating in-vivo loading conditions, but again is incapable of fully characterizing multi-axial behaviour. It can provide a better estimate than uniaxial testing and either membrane bulge testing or a tethered planar configuration have the advantage of being suitable for tissue when whole vessels are unobtainable. Inflation testing provides the best simulation of in-vivo geometry and loading conditions, and while early studies dealt with samples by assuming idealized geometries and homogenous material properties, the vast range of arterial samples with irregular geometries or heterogeneous properties due to active remodelling processes or pathological conditions 
has led to the development of three-dimensional whole-body imaging systems better equipped to deal with such vessels. These full-field measurements allow for far greater flexibility and provide a wealth of data, as well as providing more reliable information.

While continuing advances made in the development of inverse methods and emerging methods for determining material properties in-vivo [8] potentially allow constitutive equations to be formulated without recourse to in-vitro testing; in-vitro test regimes offer a significant advantage in their ability to precisely control their mechanical environment as well as allowing for testing until failure. While the stress in many clinical scenarios can be accurately simulated without dependency on material constitutive properties [166], knowledge of material yield strength or damage mechanism at high stresses is necessary, in particular with abdominal aortic aneurysm rupture prediction [5] or modelling arterial damage in balloon angioplasty [3] or arterial clamping [2].

With the influence of mechanobiological processes becoming better understood, [187] invitro mechanical testing can provide a unique advantage in isolating the role of mechanical loading on vascular remodelling processes via the use of bioreactor systems combined with inflation testing techniques. As experimental techniques turn towards systems capable of fullfield measurements of complex geometries, there remains a need for developing and improving the accuracy and robustness of these measurement techniques. The analysis and characterisation of heterogeneous, anisotropic and irregular samples can produce and inform more accurate surgical simulations, as well aiding in the risk prediction of pathological conditions. 
1. World Health Organization, World Health Statistics 2012, 2012.

2. Gasser, T.C., C.A. Schulze-Bauer, and G.A. Holzapfel, A three-dimensional finite element model for arterial clamping. J Biomech Eng, 2002. 124(4): p. 355-63.

3. Balzani, D., S. Brinkhues, and G.A. Holzapfel, Constitutive framework for the modeling of damage in collagenous soft tissues with application to arterial walls. Computer Methods in Applied Mechanics and Engineering, 2012. 213-216: p. 139-151.

4. Pierce, D.M., et al., Human thoracic and abdominal aortic aneurysmal tissues: Damage experiments, statistical analysis and constitutive modeling. Journal of the Mechanical Behavior of Biomedical Materials, 2015. 41: p. 92-107.

5. Vande Geest, J.P., et al., Towards a noninvasive method for determination of patient-specific wall strength distribution in abdominal aortic aneurysms. Ann Biomed Eng, 2006. 34(7): p. 1098-106.

6. Weisbecker, H., et al., Layer-specific damage experiments and modeling of human thoracic and abdominal aortas with non-atherosclerotic intimal thickening. J Mech Behav Biomed Mater, 2012. 12: p. 93-106.

7. Han, H.-C., et al., Artery Buckling: New Phenotypes, Models, and Applications. Annals of biomedical engineering, 2013. 41(7): $p$. 1399-1410.

8. Franquet, $A$., et al., Identification of the in vivo elastic properties of common carotid arteries from MRI: A study on subjects with and without atherosclerosis. Journal of the Mechanical Behavior of Biomedical Materials, 2013. 27: p. 184-203.

9. Schulze-Bauer, C.A., C. Morth, and G.A. Holzapfel, Passive biaxial mechanical response of aged human iliac arteries. J Biomech Eng, 2003. 125(3): p. 395-406.

10. Velican, C. and D. Velican, Study of coronary intimal thickening. Atherosclerosis, 1985. 56(3): p. 331-44.

11. Canham, P.B., et al., Measurements from light and polarised light microscopy of human coronary arteries fixed at distending pressure. Cardiovasc Res, 1989. 23(11): p. 973-82.

12. Rhodin, J.A.G., Architecture of the Vessel Wall, in Comprehensive Physiology. 2011, John Wiley \& Sons, Inc.

13. Holzapfel, G.A., et al., Determination of layer-specific mechanical properties of human coronary arteries with nonatherosclerotic intimal thickening and related constitutive modeling. American Journal of Physiology - Heart and Circulatory Physiology, 2005. 289(5): $p$. H2O48-H2O58.

14. Yu, Q., J. Zhou, and Y.C. Fung, Neutral axis location in bending and Young's modulus of different layers of arterial wall. American Journal of Physiology - Heart and Circulatory Physiology, 1993. 265(1): p. H52-H60.

15. Hasenfuss, G., Animal models of human cardiovascular disease, heart failure and hypertrophy. Cardiovasc Res, 1998. 39(1): p. 60-76.

16. Russell, J.C. and S.D. Proctor, Small animal models of cardiovascular disease: tools for the study of the roles of metabolic syndrome, dyslipidemia, and atherosclerosis. Cardiovasc Pathol, 2006. 15(6): p. 318-30.

17. Zaragoza, C., et al., Animal models of cardiovascular diseases. J Biomed Biotechnol, 2011. 2011: p. 497841.

18. Han, H.-C. and Y.-C. Fung, Longitudinal strain of canine and porcine aortas. Journal of Biomechanics, 1995. 28(5): p. 637-641.

19. Reece, W.O., (Ed.), Duke's Physiology of Domestic Animals, Twelth Edition. 12th ed. 2004, Sage House, 512 East State Street, Ithaca, New York 14850: Cornell University Press.

20. Learoyd, B.M. and M.G. Taylor, Alterations with age in the viscoelastic properties of human arterial walls. Circ Res, 1966. 18(3): p. 278-92.

21. Delfino, A., et al., Residual strain effects on the stress field in a thick wall finite element model of the human carotid bifurcation. Journal of Biomechanics, 1997. 30(8): p. 777-786. 
22. Mesangeau, D., D. Laude, and J.L. Elghozi, Early detection of cardiovascular autonomic neuropathy in diabetic pigs using blood pressure and heart rate variability. Cardiovasc Res, 2000. 45(4): p. 889-99.

23. Han, H.-C. and D. Ku, Contractile Responses in Arteries Subjected to Hypertensive Pressure in Seven-Day Organ Culture. Annals of Biomedical Engineering, 2001. 29(6): p. 467-475.

24. Rachev, A. and T. Shazly, A Preliminary Analysis of the Data From an in Vitro InflationExtension Test Can Validate the Assumption of Arterial Tissue Elasticity. Journal of Biomechanical Engineering, 2013. 135(8): p. 084502-084502.

25. Hoglund, K., et al., Blood pressure, heart rate, and urinary catecholamines in healthy dogs subjected to different clinical settings. J Vet Intern Med, 2012. 26(6): p. 1300-8.

26. Holzapfel, G., T. Gasser, and R. Ogden, A New Constitutive Framework for Arterial Wall Mechanics and a Comparative Study of Material Models. Journal of elasticity and the physical science of solids, 2000. 61(1-3): p. 1-48.

27. Carli, G., Blood pressure and heart rate in the rabbit during animal hypnosis. Electroencephalogr Clin Neurophysiol, 1974. 37(3): p. 231-7.

28. Matsumoto, T., et al., Mechanical and dimensional adaptation of rabbit carotid artery cultured in vitro. Med Biol Eng Comput, 1999. 37(2): p. 252-6.

29. Guo, X. and G.S. Kassab, Variation of mechanical properties along the length of the aorta in C57bl/6 mice. Am J Physiol Heart Circ Physiol, 2003. 285(6): p. H2614-22.

30. Gleason, R.L., et al., A multiaxial computer-controlled organ culture and biomechanical device for mouse carotid arteries. J Biomech Eng, 2004. 126(6): p. 787-95.

31. Dye, W.W., et al., Altered biomechanical properties of carotid arteries in two mouse models of muscular dystrophy. J Appl Physiol (1985), 2007. 103(2): p. 664-72.

32. Adham, M., et al., Mechanical characteristics of fresh and frozen human descending thoracic aorta. J Surg Res, 1996. 64(1): p. 32-4.

33. Stemper, B.D., et al., Mechanics of fresh, refrigerated, and frozen arterial tissue. J Surg Res, 2007. 139(2): p. 236-42.

34. Ebenstein, D.M., et al., Nanomechanical properties of calcification, fibrous tissue, and hematoma from atherosclerotic plaques. Journal of Biomedical Materials Research Part A, 2009. 91A(4): p. 1028-1037.

35. Armentano, R.L., et al., An in vitro study of cryopreserved and fresh human arteries: a comparison with ePTFE prostheses and human arteries studied non-invasively in vivo. Cryobiology, 2006. 52(1): p. 17-26.

36. Bia, D., et al., Cryopreservation procedure does not modify human carotid homografts mechanical properties: an isobaric and dynamic analysis. Cell Tissue Bank, 2006. 7(3): p. 18394.

37. Gournier, J.-P., et al., Cryopreserved arterial homografts: Preliminary study. Annals of Vascular Surgery, 1993. 7(6): p. 503-511.

38. Pukacki, F., et al., The mechanical properties of fresh and cryopreserved arterial homografts. Eur J Vasc Endovasc Surg, 2000. 20(1): p. 21-4.

39. Santana, D.B., et al., Functional properties of fresh and cryopreserved carotid and femoral arteries, and of venous and synthetic grafts: comparison with arteries from normotensive and hypertensive patients. Cell Tissue Bank, 2007. 8(1): p. 43-57.

40. Chow, M.J. and Y. Zhang, Changes in the mechanical and biochemical properties of aortic tissue due to cold storage. J Surg Res, 2011. 171(2): p. 434-42.

41. Rosset, E., et al., Effects of cryopreservation on the viscoelastic properties of human arteries. Ann Vasc Surg, 1996. 10(3): p. 262-72.

42. Venkatasubramanian, R.T., et al., Effects of freezing and cryopreservation on the mechanical properties of arteries. Ann Biomed Eng, 2006. 34(5): p. 823-32. 
43. O'Leary, S.A., B.J. Doyle, and T.M. McGloughlin, The impact of long term freezing on the mechanical properties of porcine aortic tissue. J Mech Behav Biomed Mater, 2014. 37: $p$. 165-73.

44. García-Herrera, C.M., et al., Mechanical behaviour and rupture of normal and pathological human ascending aortic wall. Medical \& Biological Engineering \& Computing, 2012. 50(6): $p$. 559-566.

45. Iliopoulos, D.C., et al., Regional and directional variations in the mechanical properties of ascending thoracic aortic aneurysms. Medical Engineering \& Physics, 2009. 31(1): p. 1-9.

46. Iliopoulos, D.C., et al., Ascending thoracic aortic aneurysms are associated with compositional remodeling and vessel stiffening but not weakening in age-matched subjects. The Journal of Thoracic and Cardiovascular Surgery, 2009. 137(1): p. 101-109.

47. Pham, T., et al., Biomechanical characterization of ascending aortic aneurysm with concomitant bicuspid aortic valve and bovine aortic arch. Acta Biomaterialia, 2013. 9(8): $p$. 7927-7936.

48. Fung, Y.C., Elasticity of soft tissues in simple elongation. Am J Physiol, 1967. 213(6): p. 153244.

49. Guinea, G.V., et al., Thermo-Mechanical Behaviour of Human Carotid Arteries in the Passive State. American Journal of Physiology - Heart and Circulatory Physiology, 2005.

50. Humphrey, J.D., Continuum thermomechanics and the clinical treatment of disease and injury. Applied Mechanics Reviews, 2003. 56(2): p. 231-260.

51. Humphrey, A.J.D. and R.M. Epstein, Cardiovascular Solid Mechanics: Cells, Tissues, and Organs. Applied Mechanics Reviews, 2002. 55(5): p. B103-B104.

52. Carew, E.O., J.E. Barber, and I. Vesely, Role of Preconditioning and Recovery Time in Repeated Testing of Aortic Valve Tissues: Validation Through Quasilinear Viscoelastic Theory. Annals of Biomedical Engineering, 2000. 28(9): p. 1093-1100.

53. Cheng, S., E.C. Clarke, and L.E. Bilston, The effects of preconditioning strain on measured tissue properties. J Biomech, 2009. 42(9): p. 1360-2.

54. Sacks, M., Biaxial Mechanical Evaluation of Planar Biological Materials. Journal of elasticity and the physical science of solids, 2000. 61(1-3): p. 199-246.

55. Lanir, Y. and Y.C. Fung, Two-dimensional mechanical properties of rabbit skin. II. Experimental results. J Biomech, 1974. 7(2): p. 171-82.

56. Eshel, $H$. and $Y$. Lanir, Effects of strain level and proteoglycan depletion on preconditioning and viscoelastic responses of rat dorsal skin. Ann Biomed Eng, 2001. 29(2): p. 164-72.

57. Choudhury, N., et al., Local mechanical and structural properties of healthy and diseased human ascending aorta tissue. Cardiovascular Pathology, 2009. 18(2): p. 83-91.

58. O'Leary, S.A., B.J. Doyle, and T.M. McGloughlin, Comparison of methods used to measure the thickness of soft tissues and their influence on the evaluation of tensile stress. Journal of Biomechanics, 2013. 46(11): p. 1955-1960.

59. Okamoto, R.J., et al., Mechanical properties of dilated human ascending aorta. Ann Biomed Eng, 2002. 30(5): p. 624-35.

60. Vorp, D.A., et al., Effect of aneurysm on the tensile strength and biomechanical behavior of the ascending thoracic aorta. The Annals of Thoracic Surgery, 2003. 75(4): p. 1210-1214.

61. Wan, W., J.B. Dixon, and R.L. Gleason, Jr., Constitutive modeling of mouse carotid arteries using experimentally measured microstructural parameters. Biophys J, 2012. 102(12): $p$. 2916-25.

62. Schriefl, A.J., et al., Quantitative assessment of collagen fibre orientations from twodimensional images of soft biological tissues. Journal of The Royal Society Interface, 2012. 9(76): p. 3081-3093.

63. Agianniotis, A. and N. Stergiopulos, Wall properties of the apolipoprotein E-deficient mouse aorta. Atherosclerosis, 2012. 223(2): p. 314-320. 
64. Lee, A.Y., et al., Effects of elastin degradation and surrounding matrix support on artery stability. American Journal of Physiology - Heart and Circulatory Physiology, 2012. 302(4): $p$. H873-H884.

65. Tong, J., et al., Effects of Age on the Elastic Properties of the Intraluminal Thrombus and the Thrombus-covered Wall in Abdominal Aortic Aneurysms: Biaxial Extension Behaviour and Material Modelling. European Journal of Vascular and Endovascular Surgery, 2011. 42(2): $p$. 207-219.

66. Vorp, D.A., et al., Association of intraluminal thrombus in abdominal aortic aneurysm with local hypoxia and wall weakening. Journal of Vascular Surgery, 2001. 34(2): p. 291-299.

67. Heiland, V.M., et al., Identification of carotid plaque tissue properties using an experimentalnumerical approach. Journal of the Mechanical Behavior of Biomedical Materials, 2013. 27: p. 226-238.

68. Davis, N.P., et al., Sustained axial loading lengthens arteries in organ culture. Ann Biomed Eng, 2005. 33(7): p. 867-77.

69. Han, H.C., D.N. Ku, and R.P. Vito, Arterial wall adaptation under elevated longitudinal stretch in organ culture. Ann Biomed Eng, 2003. 31(4): p. 403-11.

70. Nichol, J., et al., Hemodynamic Conditions Alter Axial and Circumferential Remodeling of Arteries Engineered Ex Vivo. Annals of Biomedical Engineering, 2005. 33(6): p. 721-732.

71. Wayman, B.H., et al., Arteries respond to independent control of circumferential and shear stress in organ culture. Ann Biomed Eng, 2008. 36(5): p. 673-84.

72. O'Leary, S.A., et al., Determining the influence of calcification on the failure properties of abdominal aortic aneurysm (AAA) tissue. Journal of the Mechanical Behavior of Biomedical Materials, 2015. 42: p. 154-167.

73. Sommer, G., et al., Dissection properties of the human aortic media: an experimental study. J Biomech Eng, 2008. 130(2): p. 021007.

74. Holzapfel, G.A. and R.W. Ogden, Constitutive modelling of arteries. Proceedings of the Royal Society of London A: Mathematical, Physical and Engineering Sciences, 2010. 466(2118): $p$. 1551-1597.

75. Humphrey, J.D., Mechanics of the arterial wall: review and directions. Crit Rev Biomed Eng, 1995. 23(1-2): p. 1-162.

76. ASTM International, ASTM D412-06a(2013), in Standard Test Methods for Vulcanized Rubber and Thermoplastic Elastomers-Tension2013, ASTM International: West Conshohocken, PA.

77. Davis, J.R., Tensile Testing, Second Edition. Second Edition ed. 2004, Materials Park, Ohio 44073-0002: ASM International.

78. Ng, B.H., S.M. Chou, and V. Krishna, The influence of gripping techniques on the tensile properties of tendons. Proc Inst Mech Eng H, 2005. 219(5): p. 349-54.

79. Mulvihill, J.J., et al., Mechanical, biological and structural characterization of in vitro ruptured human carotid plaque tissue. Acta Biomater, 2013. 9(11): p. 9027-35.

80. Shazly, T., et al., On the Uniaxial Ring Test of Tissue Engineered Constructs. Experimental Mechanics, 2015. 55(1): p. 41-51.

81. Zhang, D. and D.D. Arola, Applications of digital image correlation to biological tissues. J Biomed Opt, 2004. 9(4): p. 691-9.

82. Zhang, D., C. Eggleton, and D. Arola, Evaluating the mechanical behavior of arterial tissue using digital image correlation. Experimental Mechanics, 2002. 42(4): p. 409-416.

83. Chuong, C.J. and Y.C. Fung, Compressibility and constitutive equation of arterial wall in radial compression experiments. J Biomech, 1984. 17(1): p. 35-40.

84. Miller, K., How to test very soft biological tissues in extension? Journal of Biomechanics, 2001. 34(5): p. 651-657.

85. Morriss, L., A. Wittek, and K. Miller, Compression testing of very soft biological tissues using semi-confined configuration-A word of caution. Journal of biomechanics, 2008. 41(1): $p$. 235-238. 
86. Cox, R.H., Comparison of arterial wall mechanics using ring and cylindrical segments. Am J Physiol, 1983. 244(2): p. H298-303.

87. Stoiber, M., et al., A method for mechanical characterization of small blood vessels and vascular grafts. Experimental Mechanics, 2015: p. 1-5.

88. Seliktar, D., et al., Dynamic Mechanical Conditioning of Collagen-Gel Blood Vessel Constructs Induces Remodeling In Vitro. Annals of Biomedical Engineering, 2000. 28(4): p. 351-362.

89. Chuong, C.J. and Y.C. Fung, On Residual Stresses in Arteries. Journal of Biomechanical Engineering, 1986. 108(2): p. 189-192.

90. Vaishnav, R.N., J. Vossoughi, and C.W. Hall, Estimation of Residual Strains in Aortic Segments, in Biomedical Engineering II. 1983, Pergamon. p. 330-333.

91. Chaudhry, H.R., et al., Residual stresses in oscillating thoracic arteries reduce circumferential stresses and stress gradients. Journal of Biomechanics, 1997. 30(1): p. 57-62.

92. Takamizawa, $K$. and $K$. Hayashi, Strain energy density function and uniform strain hypothesis for arterial mechanics. J Biomech, 1987. 20(1): p. 7-17.

93. Zhang, $W$., et al., The effect of longitudinal pre-stretch and radial constraint on the stress distribution in the vessel wall: a new hypothesis. Mech Chem Biosyst, 2005. 2(1): p. 41-52.

94. Labrosse, M.R., et al., Mechanical characterization of human aortas from pressurization testing and a paradigm shift for circumferential residual stress. Journal of the Mechanical Behavior of Biomedical Materials, 2013. 17: p. 44-55.

95. Liu, S.Q. and Y.C. Fung, Relationship Between Hypertension, Hypertrophy, and Opening Angle of Zero-Stress State of Arteries Following Aortic Constriction. Journal of Biomechanical Engineering, 1989. 111(4): p. 325-335.

96. Han, H.C. and Y.C. Fung, Direct measurement of transverse residual strains in aorta. American Journal of Physiology - Heart and Circulatory Physiology, 1996. 270(2): p. H750H759.

97. Rachev, A. and S.E. Greenwald, Residual strains in conduit arteries. Journal of Biomechanics, 2003. 36(5): p. 661-670.

98. Guo, X., et al., Morphometry and strain distribution of the C57BL/6 mouse aorta. Am J Physiol Heart Circ Physiol, 2002. 283(5): p. H1829-37.

99. Zhao, J., et al., Regional arterial stress-strain distributions referenced to the zero-stress state in the rat. Am J Physiol Heart Circ Physiol, 2002. 282(2): p. H622-9.

100. Fung, Y.C. and S.Q. Liu, Change of residual strains in arteries due to hypertrophy caused by aortic constriction. Circ Res, 1989. 65(5): p. 1340-9.

101. Wang, R. and R.L. Gleason, A Mechanical Analysis of Conduit Arteries Accounting for Longitudinal Residual Strains. Annals of Biomedical Engineering, 2010. 38(4): p. 1377-1387.

102. Holzapfel, G., et al., Layer-Specific 3D Residual Deformations of Human Aortas with NonAtherosclerotic Intimal Thickening. Annals of Biomedical Engineering, 2007. 35(4): p. 530545.

103. Holzapfel, G.A. and R.W. Ogden, Modelling the layer-specific three-dimensional residual stresses in arteries, with an application to the human aorta. Journal of the Royal Society Interface, 2010. 7(46): p. 787-799.

104. Pierce, D.M., et al., A method for incorporating three-dimensional residual stretches/stresses into patient-specific finite element simulations of arteries. J Mech Behav Biomed Mater, 2015. 47: p. 147-64.

105. Carnell, P., R. Vito, and W.R. Taylor, Characterizing intramural stress and inflammation in hypertensive arterial bifurcations. Biomechanics and Modeling in Mechanobiology, 2007. 6(6): p. 409-421.

106. Sommer, G., et al., Multiaxial mechanical properties and constitutive modeling of human adipose tissue: $A$ basis for preoperative simulations in plastic and reconstructive surgery. Acta Biomaterialia, 2013. 9(11): p. 9036-9048. 
107. Azadani, A.N., et al., Comparison of Mechanical Properties of Human Ascending Aorta and Aortic Sinuses. The Annals of Thoracic Surgery, 2012. 93(1): p. 87-94.

108. Kamenskiy, A.V., et al., Passive biaxial mechanical properties and in vivo axial pre-stretch of the diseased human femoropopliteal and tibial arteries. Acta Biomaterialia, 2014. 10(3): $p$. 1301-1313.

109. O'Leary, S.A., B.J. Doyle, and T.M. McGloughlin, The impact of long term freezing on the mechanical properties of porcine aortic tissue. Journal of the Mechanical Behavior of Biomedical Materials, 2014. 37(0): p. 165-173.

110. Vande Geest, J.P., M.S. Sacks, and D.A. Vorp, Age dependency of the biaxial biomechanical behavior of human abdominal aorta. J Biomech Eng, 2004. 126(6): p. 815-22.

111. Vande Geest, J.P., M.S. Sacks, and D.A. Vorp, The effects of aneurysm on the biaxial mechanical behavior of human abdominal aorta. Journal of Biomechanics, 2006. 39(7): $p$. 1324-1334.

112. Vande Geest, J.P., M.S. Sacks, and D.A. Vorp, A planar biaxial constitutive relation for the luminal layer of intra-luminal thrombus in abdominal aortic aneurysms. J Biomech, 2006. 39(13): p. 2347-54.

113. O'Leary, S.A., et al., The biaxial biomechanical behavior of abdominal aortic aneurysm tissue. Ann Biomed Eng, 2014. 42(12): p. 2440-50.

114. O'Leary, S.A., et al., The biaxial mechanical behaviour of abdominal aortic aneurysm intraluminal thrombus: classification of morphology and the determination of layer and region specific properties. J Biomech, 2014. 47(6): p. 1430-7.

115. Sun, W., M.S. Sacks, and M.J. Scott, Effects of Boundary Conditions on the Estimation of the Planar Biaxial Mechanical Properties of Soft Tissues. Journal of Biomechanical Engineering, 2005. 127(4): p. 709-715.

116. O'Leary, S.A., et al., The biaxial mechanical behaviour of abdominal aortic aneurysm intraluminal thrombus: Classification of morphology and the determination of layer and region specific properties. Journal of Biomechanics, 2014. 47(6): p. 1430-1437.

117. Sun, W., et al., Biaxial Mechanical Response of Bioprosthetic Heart Valve Biomaterials to High In-plane Shear. Journal of Biomechanical Engineering, 2003. 125(3): p. 372-380.

118. Hardy, W.N., et al., Study of potential mechanisms of traumatic rupture of the aorta using insitu experiments. Stapp Car Crash J, 2006. 50: p. 247-66.

119. Shah, C.S., et al., Dynamic biaxial tissue properties of the human cadaver aorta. Stapp Car Crash J, 2006. 50: p. 217-46.

120. Mohan, D. and J.W. Melvin, Failure properties of passive human aortic tissue. I--uniaxial tension tests. J Biomech, 1982. 15(11): p. 887-902.

121. Eilaghi, A., et al., Strain Uniformity in Biaxial Specimens is Highly Sensitive to Attachment Details. Journal of Biomechanical Engineering, 2009. 131(9): p. $091003-091003$.

122. Nielsen, P.M.F., P.J. Hunter, and B.H. Smaill, Biaxial Testing of Membrane Biomaterials: Testing Equipment and Procedures. Journal of Biomechanical Engineering, 1991. 113(3): $p$. 295-300.

123. Ramault, C., et al., Comparison of Different Techniques for Strain Monitoring of a Biaxially Loaded Cruciform Specimen. Strain, 2011. 47: p. 210-217.

124. Holzapfel, G.A. and R.W. Ogden, On planar biaxial tests for anisotropic nonlinearly elastic solids. A continuum mechanical framework. Mathematics and Mechanics of Solids, 2009(14): p. 474-489.

125. Sommer, G., et al., Quantification of Shear Deformations and Corresponding Stresses in the Biaxially Tested Human Myocardium. Annals of Biomedical Engineering, 2015. 43(10): $p$. 2334-2348.

126. Zhang, W., et al., A generalized method for the analysis of planar biaxial mechanical data using tethered testing configurations. J Biomech Eng, 2015. 137(6): p. 064501. 
127. Humphrey, J.D., et al., Computer-aided vascular experimentation: a new electromechanical test system. Ann Biomed Eng, 1993. 21(1): p. 33-43.

128. Doyle, B.J., et al., Identification of rupture locations in patient-specific abdominal aortic aneurysms using experimental and computational techniques. Journal of Biomechanics, 2010. 43(7): p. 1408-1416.

129. Vorp, D.A., et al., A device for the application of cyclic twist and extension on perfused vascular segments. Am J Physiol, 1996. 270(2 Pt 2): p. H787-95.

130. Liu, Q. and H.-C. Han, Mechanical Buckling of Artery under Pulsatile Pressure. Journal of Biomechanics, 2012. 45(7): p. 1192-1198.

131. Schulze-Bauer, C.A.J. and G.A. Holzapfel, Determination of constitutive equations for human arteries from clinical data. Journal of Biomechanics, 2003. 36(2): p. 165-169.

132. Blondel, W.C.P.M., et al., Rheological properties of fresh and cryopreserved human arteries tested in vitro. Rheologica Acta, 2000. 39(5): p. 461-468.

133. Guo, X. and G.S. Kassab, Variation of mechanical properties along the length of the aorta in C57bl/6 mice. Vol. 285. 2003. H2614-H2622.

134. Saravanan, U., et al., On the Deformation of the Circumflex Coronary Artery During Inflation Tests at Constant Length. Experimental Mechanics, 2006. 46(5): p. 647-656.

135. Schulze-Bauer, C.A., P. Regitnig, and G.A. Holzapfel, Mechanics of the human femoral adventitia including the high-pressure response. Am J Physiol Heart Circ Physiol, 2002. 282(6): p. H2427-40.

136. Stergiopulos, N., et al., Assessing the homogeneity of the elastic properties and composition of the pig aortic media. J Vasc Res, 2001. 38(3): p. 237-46.

137. Valdez-Jasso, D., et al., Analysis of Viscoelastic Wall Properties in Ovine Arteries. Biomedical Engineering, IEEE Transactions on, 2009. 56(2): p. 210-219.

138. van den Broek, C.N., et al., A generic constitutive model for the passive porcine coronary artery. Biomech Model Mechanobiol, 2011. 10(2): p. 249-58.

139. Kim, J. and S. Baek, Circumferential variations of mechanical behavior of the porcine thoracic aorta during the inflation test. Journal of Biomechanics, 2011. 44(10): p. 1941-1947.

140. Gleason, R.L., E. Wilson, and J.D. Humphrey, Biaxial biomechanical adaptations of mouse carotid arteries cultured at altered axial extension. J Biomech, 2007. 40(4): p. 766-76.

141. Sommer, G., et al., Biaxial mechanical properties of intact and layer-dissected human carotid arteries at physiological and supraphysiological loadings. Am J Physiol Heart Circ Physiol, 2010. 298(3): p. H898-912.

142. Pao, Y.C., J.T. Lu, and E.L. Ritman, Bending and twisting of an in vivo coronary artery at a bifurcation. J Biomech, 1992. 25(3): p. 287-95.

143. Zhang, W., X. Lu, and G.S. Kassab, Shear modulus of porcine coronary artery in reference to a new strain measure. Biomaterials, 2007. 28(31): p. 4733-4738.

144. Deng, S.X., et al., New experiments on shear modulus of elasticity of arteries. Am J Physiol, 1994. 266(1 Pt 2): p. H1-10.

145. Van Epps, J.S. and D.A. Vorp, A New Three-Dimensional Exponential Material Model of the Coronary Arterial Wall to Include Shear Stress Due to Torsion. Journal of Biomechanical Engineering, 2008. 130(5): p. 051001-051001.

146. Walsh, M.T., et al., Uniaxial tensile testing approaches for characterisation of atherosclerotic plaques. Journal of Biomechanics, 2014. 47(4): p. 793-804.

147. Doyle, B.J., et al., On the influence of patient-specific material properties in computational simulations: A case study of a large ruptured abdominal aortic aneurysm. International Journal for Numerical Methods in Biomedical Engineering, 2013. 29(2): p. 150-164.

148. O'Leary, S.A., et al., Determining the influence of calcification on the failure properties of abdominal aortic aneurysm (AAA) tissue. J Mech Behav Biomed Mater, 2015. 42: p. 154-67.

149. Humphrey, J.D., Vascular Adaptation and Mechanical Homeostasis at Tissue, Cellular, and Sub-cellular Levels. Cell Biochemistry and Biophysics, 2008. 50(2): p. 53-78. 
150. Pedrigi, R.M., et al., Regional mechanical properties and stress analysis of the human anterior lens capsule. Vision Research, 2007. 47(13): $p$. 1781-1789.

151. Everett, W.N., P. Shih, and J.D. Humphrey, A bi-plane video-based system for studying the mechanics of arterial bifurcations. Experimental Mechanics, 2005. 45(4): p. 377-382.

152. Genovese, $K$. and $C$. Pappalettere, Whole 3D shape reconstruction of vascular segments under pressure via fringe projection techniques. Optics and Lasers in Engineering, 2006. 44(12): p. 1311-1323.

153. Sutton, M.A., et al., Strain field measurements on mouse carotid arteries using microscopic three-dimensional digital image correlation. J Biomed Mater Res A, 2008. 84(1): p. 178-90.

154. Genovese, K., Radial metrology application to whole-body measurement on hyperelastic tubular samples. Optics and Lasers in Engineering, 2007. 45(11): p. 1059-1066.

155. Genovese, K., A video-optical system for time-resolved whole-body measurement on vascular segments. Optics and Lasers in Engineering, 2009. 47(9): p. 995-1008.

156. Genovese, K., et al., Digital image correlation-based point-wise inverse characterization of heterogeneous material properties of gallbladder in vitro. Proceedings of the Royal Society A: Mathematical, Physical and Engineering Science, 2014. 470(2167).

157. Genovese, K. and C. Pappalettere, Axial stereo-photogrammetry for $360^{\circ}$ measurement on tubular samples. Optics and Lasers in Engineering, 2007. 45(5): p. 637-650.

158. Kim, J.H., et al., Experimental characterization of rupture in human aortic aneurysms using a full-field measurement technique. Biomech Model Mechanobiol, 2012. 11(6): p. 841-53.

159. Avril, S., et al., Overview of Identification Methods of Mechanical Parameters Based on Fullfield Measurements. Experimental Mechanics, 2008. 48(4): p. 381-402.

160. Einstein, D.R., et al., Inverse parameter fitting of biological tissues: a response surface approach. Ann Biomed Eng, 2005. 33(12): p. 1819-30.

161. Avril, S., P. Badel, and A. Duprey, Anisotropic and hyperelastic identification of in vitro human arteries from full-field optical measurements. J Biomech, 2010. 43(15): p. 2978-85.

162. Lu, J., X. Zhou, and M. Raghavan, Inverse method of stress analysis for cerebral aneurysms. Biomechanics and Modeling in Mechanobiology, 2008. 7(6): p. 477-486.

163. Lu, J., X. Zhou, and M.L. Raghavan, Inverse elastostatic stress analysis in pre-deformed biological structures: Demonstration using abdominal aortic aneurysms. Journal of Biomechanics, 2007. 40(3): p. 693-696.

164. Lu, J., X.L. Zhou, and M.L. Raghavan, Computational method of inverse elastostatics for anisotropic hyperelastic solids. International Journal for Numerical Methods in Engineering, 2007. 69(6): p. 1239-1261.

165. Joldes, G.R., A. Wittek, and K. Miller, A Total Lagrangian based method for recovering the undeformed configuration in finite elasticity. Applied Mathematical Modelling, 2015. 39(14): $p$. 3913-3923.

166. Joldes, G.R., et al., A simple, effective and clinically applicable method to compute abdominal aortic aneurysm wall stress. J Mech Behav Biomed Mater, 2015.

167. Mohan, D. and J.W. Melvin, Failure properties of passive human aortic tissue. II--Biaxial tension tests. J Biomech, 1983. 16(1): p. 31-44.

168. Marra, S.P., et al., Elastic and rupture properties of porcine aortic tissue measured using inflation testing. Cardiovasc Eng, 2006. 6(4): p. 123-31.

169. Janz, R.F., Estimation of local myocardial stress. Am J Physiol, 1982. 242(5): p. H875-81.

170. Hsu, F.P.K., et al., A triplane video-based experimental system for studying axisymmetrically inflated biomembranes. Transactions of Biomedical Engineering, 1995. 42(5): p. 442-450.

171. Romo, A., et al. Mechanical characterization of the thoracic ascending aorta. in 2012 IRCOBI Conference Proceedings - International Research Council on the Biomechanics of Injury. 2012.

172. Romo, A., et al., In vitro analysis of localized aneurysm rupture. Journal of Biomechanics, 2014. 47(3): p. 607-616. 
173. Lehoux, S., Y. Castier, and A. Tedgui, Molecular mechanisms of the vascular responses to haemodynamic forces. Journal of Internal Medicine, 2006. 259(4): p. 381-392.

174. Skalak, T.C. and R.J. Price, The Role of Mechanical Stresses in Microvascular Remodeling. Microcirculation, 1996. 3(2): p. 143-165.

175. Taber, L.A., Biomechanics of Growth, Remodeling, and Morphogenesis. Applied Mechanics Reviews, 1995. 48(8): p. 487-545.

176. De Mey, J.G., et al., Acute and long-term effects of tissue culture on contractile reactivity in renal arteries of the rat. Circ Res, 1989. 65(4): p. 1125-35.

177. Lindqvist, A., B.O. Nilsson, and P. Hellstrand, Inhibition of calcium entry preserves contractility of arterial smooth muscle in culture. J Vasc Res, 1997. 34(2): p. 103-8.

178. Bardy, N., et al., Differential effects of pressure and flow on DNA and protein synthesis and on fibronectin expression by arteries in a novel organ culture system. Circ Res, 1995. 77(4): $p$. 684-94.

179. Bolz, S.S., et al., Intact endothelial and smooth muscle function in small resistance arteries after 48 h in vessel culture. Am J Physiol Heart Circ Physiol, 2000. 279(3): p. H1434-9.

180. Clerin, V., et al., Mechanical environment, donor age, and presence of endothelium interact to modulate porcine artery viability ex vivo. Ann Biomed Eng, 2002. 30(9): p. 1117-27.

181. Clerin, V., et al., Tissue engineering of arteries by directed remodeling of intact arterial segments. Tissue Eng, 2003. 9(3): p. 461-72.

182. Guo, H., J.D. Humphrey, and M.J. Davis, Effects of biaxial stretch on arteriolar function in vitro. Am J Physiol Heart Circ Physiol, 2007. 292(5): $p$. H2378-86.

183. Lawrence, A.R. and K.J. Gooch, Differences in transmural pressure and axial loading ex vivo affect arterial remodeling and material properties. J Biomech Eng, 2009. 131(10): p. 101009.

184. Lawrence, A.R. and K.J. Gooch, Transmural pressure and axial loading interactively regulate arterial remodeling ex vivo. Am J Physiol Heart Circ Physiol, 2009. 297(1): p. H475-84.

185. Bakker, E.N., et al., Organoid culture of cannulated rat resistance arteries: effect of serum factors on vasoactivity and remodeling. Am J Physiol Heart Circ Physiol, 2000. 278(4): p. H1233-40.

186. Zaucha, M.T., et al., A novel cylindrical biaxial computer-controlled bioreactor and biomechanical testing device for vascular tissue engineering. Tissue Eng Part A, 2009. 15(11): p. 3331-40.

187. Humphrey, J.D. and G.A. Holzapfel, Mechanics, mechanobiology, and modeling of human abdominal aorta and aneurysms. Journal of Biomechanics, 2012. 45(5): p. 805-814. 